(Aus Dr. Abel's Frauenklinik in Berlin.)

\title{
Ueber Blutanhäufungen bei doppelten Genitalien mit Verschluss einer Seite.
}

Von

Dr. Rudolf Katz,

Assistent der Klinik.

(Hierzu Tafel X.)

Seit den grundlegenden Arbeiten von Kussmaul (19) und Livius Fürst (8), in denen die embryologischen Processe bei der Entwickelung der Geschlechtsorgane geschildert wurden und auf Grund sorgfältiger Studien die Entstehung von Missbildungen in diesen Organen zu erklären versucht wurde, hatte man den Missbildungen der weiblichen Genitalien grössere Aufmerksamkeit und lebhafteres Interesse zugewandt. Es geht dies unzweifelhaft aus der reichhaltigen, seitdem entstandenen, diesbezüglichen Literatur hervor, die von Jahr zu Jahr bedeutend anwächst.

Ich möchte im Folgenden einen Fall, der ins Kapitel der Blutanhäufungen bei doppelten Genitalien gehört, und der in verschiedener Hinsicht, sowohl pathologisch - anatomisch als auch bezüglich der Therapie, die hier eingeschlagen wurde, manches Interessante bietet, mittheilen.

Anamnese: Das 26jährige junge Mädchen giebt an - abgesehen von einem Icterus in ihrem 9. Lebensjahre -, bis zu seinem 14. Lebensjahre stets gesund gewesen zu sein. Die Anamnese ergiebt weiter, dass Patientin seit dem 14. Jahre stark bleichsüchtig war und oft über Mattigkeit zu klagen hatte. Gleichzeitig hatte sie Beschwerden im Leib, Drängen nach unten und besonders starken Druck auf den Mastdarm. Diese Beschwerden dauerten bis zum 18. Jahre an, bis die ersten Menses auftraten. Dieselben waren immer sehr scbwach, dauerten 
aber mit Unterbrechungen mitunter 8 Tage lang. - Am Tage vor der Henstruation stellten sich stets sehr heftige Schmerzen ein und am 2. Tage der Menstruation kamen plötzliche, ruckweise auftretende Schmerzen, sodass die Patientin bisweilen ohnmächtig umfiel. Ausserdem klagt Patientin über leichte Schmerzen während der ganzen Dauer der Menstruationszeit. Im Ganzen nahmen die Schmerzen in den letzten Jahren, in welchen die Menses alle 3 Wochen eintraten, besonders am

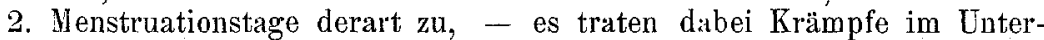
leib und Schüttelfrost auf - dass die Patientin den Zustand nicht mehr ertragen konnte und deshalb die Klinik anfsuchte.

Bei ihrer Aufnahme wurde folgender Status erhoben: Mittelgrosses, ziemlich gut genährtes Mädchen von blasser Gesichtsfarbe und mit leidendem Gesichtsausdrucke. Herz- und Lungenbefund normal. Mammae beiderseits schlecht entwickelt. - Introitus vaginae eng. Hymen intact.

Die Portio steht etwas nach links von der Führungslinie und ein wenig nach vorn. In der Verlängerung der Cervix kommt man an einen olivengrossen, stark beweglichen Uteruskörper, der etwas nach links von der Mittellinie liegt. Rechts daneben, ca. $1-2 \mathrm{~cm}$ vom Fundus entfernt, liegt ein zweiter Körper, dessen Grösse etwa dem 2. Monate der Schwangerschaft entspricht und dessen Consistenz ebenfalls die eines schwangeren Uterus ist. Beim Stossen an die Portio bewegt sich der links gelegene Uterus, der rechte Tumor dagegen nicht. Das ganze hintere Scheidengewölbe und Becken wird durch eine kindskopfgrosse Geschwulst ausgefüllt, die auf Druck äusserst schmerzhaft ist. - Die linken Adnexe können nicht besonders abgetastet werden.

Die Diagnose lautete auf: Haematometra im rechten atretischen Nebenborn eines Uterus duplex, Haematovarium (?).

Aus Gründen, auf die ich später des Näheren eingehen möchte, wurde die vaginale Koeliotomie beschlossen.

Die Operation, die am 19.6. 1901 von Dr. Abel ausgeführt wurde, ging folgendermaassen von statten: In Chloroformnarkose wurde die vordere Scheidenwand durch einen Längsschnitt eröffnet, die Blase stumpf abgesehoben, die Plica vesico-uterina eröffnet und mit einem Faden fixirt. Unter Höherklettern mit Muzeux's wird hierauf der Uterus vor die Vulva gezogen, ebenso der rechts vom Uterus sitzende, mit diesem durch Bindegewebe und Muskulatur verbundene grössere Tumor (Haematometra). Darauf - konnte der Operateur, indem er mit dem Finger hinter diesem Tumor in der Verlängerung der von ihm abgehenden Tube weiterging, auch den zweiten kindskopfgrossen, prall gefülltẹn, fluctuirenden Tumor in die Peritonealöffnung einstellen und mit einer Kornzange einstossen; es entleerte sich eine beträchtliche Menge chokoladenbrauner Flüssigkeit. Darauf wurde die Sackwand weiter herausgezogen, so dass jetzt die sämmtlichen Theile vor der Vulva waren. Es wird nunmehr zu der Exstirpation des atretischen Horns geschritten, indem der Operateur, am Fundus uteri beginnend, die Uteruskante successive mit ein paar grossen Nähten umstach (und zwar wurden die bindegewebigen und muskulösen Partien, die zwischen der Haematometra und dem linken Uterustheil lagen, dicht am Uterus abgebunden). - der Operateur hatte nun Platz und konnte die Tube, das Ligamentum infundibulo-pelvicum und ebensu das Ligamentnm latum ohne Schwierigkeit abbinden und abschneiden. - Darauf wurde sorgfältig die Uterus. 
serosa an der Uteruskante vereinigt, und der Uterus mit den gesunden linken Adnexen wieder in die Bauchhöhle zurückgebracht. Die Scheidenwunde wird in üblicher Weise geschlossen.

Der. Heilungsverlauf war absolut ungestört. Pat. hatte niemals eine Temperatursteigerung. Am 21. Tage nach der Operation steht die Pat. auf und verlässt am 29. Tage die Klinik beschwerdefrei.

1/2 Jahr nach der Operation stellt sich Pat. vor und giebt an, ganz regelmässig die Menses ohne die geringsten Beschwerden zu haben. - Nur die beiden ersten Male nach der Operation hat sie cin geringes Ziehen im Leibe während der Menstruation verspürt.

Im Uebrigen "fängt sie erst jetzt an zu leben". - Der objective Befund ist ebenfalls ein absolut guter, der Uterus liegt in Antefiexionsstellung, die Umgebung des Uterus ist normal.

\section{Beschreibung des Präparats.}

Die exstirpirten Theile wurden nach der bekannten Methode von Kajserling-Pick in natürlichen Farben conservirt und zeigen folgendes Bild (cf. Tafel X).

Der mediale Tumor (rudimentäres Nebenhorn) ist von birnförmiger Gestalt, $7 \mathrm{~cm}$ lang, $4 \mathrm{~cm}$ dick, $5 \mathrm{~cm}$ breit. An der medialen Wand ist die den Tumor bedeckende Serosa eingerissen (Verbindungsstelle mit dem andern Uterus), der Tumor ist prall gefüllt und zeigt Fluctuation. - - In dem Uterushorn fand sich eine nach unten vollständig abgeschlossene Höhle, welche mit altem, dicklichem Blute von theerartiger Beschaffenheit (21/2 Esslöffel voll) angefüllt ist. Das Horn ist mit einer blassen Mucosa ausgekleidet und enthält an seinem oberen Theile ein erbsengrosses, breitbasig aufsitzendes, in das Cavum prominirendes Gebilde. Die Wandungen des Nebenhorns sind sehr stark ausgebildet und repräsentiren auf der ganzen Peripherie ein $1 \frac{1}{2} \mathrm{~cm}$ starkes Muskellager. -- Von der lateralen Wand gehen zwei Stränge ab, der eine (untere) ist das von dem Uterus unmittelbar ausgehende Ovarium, dasselbe ist $7 \mathrm{~cm}$ lang, $1 \frac{1}{2}$ breit. Er zeigt auf seiner Oberfläche einzelne Einkerbungen und zahlreiche, erbsengrosse, blau-durchschimmernde, leicht prominirende Gebilde. Der andere (obere) Strang, der unmittelbar daneben vom Uterus entspringend, parallel verläuft, ist die Tube. Dieselbe hat vom Ostium uterinum (das im rudimentären Horn makroskopisch sichtbar ist) an bis zum muthmasslichen abdominalen Ende, von dessen Fimbrien nichts mehr zu sehen ist, eine Länge von $15 \mathrm{~cm}$. Sie ist im allgemeinen wenig geschlängelt, verläuft zunächst eine Strecke von $7 \mathrm{~cm}$ in Kleinfingerstärke, um dann in einen etwas nach oben abgeknickten oystiochen Tumor 
(Haematosalpinxsack, der in gefülltem Zustande die Grösse eines kleinen Kindskopfes hatte), überzugehen. Er ist jetzt nach Ausfliessen des grössten Theiles seines Inhaltes während der Operation, einer chocoladenfarbigen, leicht krümlichen Flüssigkeit, zusammengesunken und ist noch kleinfaustgross. Die Oberfläche des Tumorsackes, dessen Wand durchschnittlich $1 / 2 \mathrm{~cm}$ dick ist, ist unregelmässig gewulstet, dunkelblauroth und lässt zahlreiche Gefässe durchscheinen. An der Innenfläche des Sackes findet sich ein förmlicher Ueberzug einer braunröthlichen, feinkrümeligen Masse, die sich unschwer als Residuum der ursprünglichen Blutansammlung in der Tube erkennen lässt. Was den übrigen (ampullären) Theil der Tube betrifft, so zeigt sich auf den verschiedenen zur Tubenachse senkrechten Einschnitten das Lumen erweitert, mit rostbraunem, flüssigen Blute ausgefüllt, die Schleimhaut gleichfalls rostbraun, Myo- und Perisalpingium schwielig verdickt, überall und namentlich nach dem abdominalen Ende hin mehr oder minder von kleinen gelblichen oder bräunlichen Pigmentfleckchen durchsetzt. Die Tube (sowohl der nicht ausgedehnte Theil als auch der Haematosalpinxsack ist vom Ovarium durch eine Membran (ligam. latum) getrennt, die ebenfalls eine derbschwartige Verdickung zeigt. Ferner sind sowohl an der Oberfläche des Nebenhorns, an dem schwieligen Perisalpingium and an der Oberfläche des Eierstocks feinere und derbere peritonitische Stränge und Membranen zu sehen.

Von den Ergebnissen der eingehenden mikroskopischen Untersuchung (Härtung in 4 proc. Formalin, Celloidineinbettung zum Theil auch Paraffineinbettung; Färbung mit Haemalaun; Haemalaun-Eosin; nach van Gieson) heben wir Folgendes hervor: Zur mikroskopischen Untersuchung der Wand des rudimentären Hornes wurden Schnitte senkrecht zur Längsachse angelegt, und zwar von verschiedenen Stellen. Die Wandung der Höhle ist jnnen überall mit Schleimhaut bekleidet, welche wenigstens der Hauptsache nach, der gewöhnlichen Uterusschleimhaut gleicht, da es alle Kriterien einer solchen, Oberflächenepithel, Drüsen und Interglandulärsubstanz aufweist. Das Oberflächenepithel zeigt von dem der normalen Uterusschleimhaut insofern eine Abweichung, als man nirgends cylindrische Zellen erkennen kann, sondern an den wenigen Stellen, wo das Epithel erhalten ist, sind die Zellen mehr kubisch und abgeplattet, sie behalten jedoch ihre epitheliale Natur und sind deutlich gegen das darunter liegende interglanduläre 
Gewebe abgegrenzt; an anderen Stellen ist das Epithel gänzlich von dem interglandulären Gewebe abgehoben, es finden sich jedoch auch Strecken, wo diese Zellen gänzlich die Natur von Epithelzellen verloren haben und gleichsam proliferirende Zellenmassen bilden, welche ohne bestimmte Grenze in das interglanduläre Gewebe übergehen, von dessen Zellenelementen sie kaum zu unterscheiden sind.

Das interglanduläre Gewebe enthält überall, sowohl nach der Oberfläche als auch nach der Musculatur hin, reichliche kleinzellige Infiltration. Die Drüsen sind sehr zahlreich und zeigen auf ihren Durchschnitten nirgends die runde Form der normalen Uterindrüsen, sondern sind alle stark gesshlängelt und erweitert, sodass in den zur Oberfläche senkrechten Schnitten grössere und kleinere, von einer einfachen Epithelschicht bekleidete ovale Drüsenlumina zu sehen sind. Den Drüsen ist ein Cylinderepithel mit centralem Kern gemeinsam, das sich besser als das Oberflächenepithel erhalten hat und eine vollständig normale Beschaffenheit zeigt und auch den ihm eigenthümlichen Saum an der freien Oberfläche deutlich erkennen lässt. Blut ist innerhalb der Drüsen nirgends anzutreffen. Die Tunica propria der Drüsen besteht aus einer Lamelle zarten Bindegewebes mit meist spindelförmigen, abgeplatteten Zellen.

Es zeigen also die Schnitte überall das typische Bild einer interstitiellen und glandulären (und zwar hypertrophica et hyperplastica) Endometritis. Das im oberen Theil des rudimentären Horns sitzende erbsengrosses Gebilde erweist sich mikroskopisch als ein Schleimhantpolyp, dessen Oberflächenepithel gleich dem im übrigen Cavum des Horns ist und dessen histologischer Bau vollkommen der Schleimhant des Horns gleicht.

Mit ziemlich scharfer Grenze differenzirt sich die Schleimhaut von der Musculatur. Dieselbe zeigt im allgemeinen dieselben Verhältnisse wie im normalen Uterus; unmittelbar unter der Schleimhaut überwiegen die longitudinalen Faserzüge, durchsetzt von einzelnen quer verlaufenden Bündeln, allmählich beherrschen diese letzteren fast ausschliesslich das Bild, bis an der peritonealen Seite wieder die longitudinalen Fasern vorwiegen. Die äussere Muskelfaserschicht zeigt im Gegensatz zu den anderen einen verhältnissmässig grossen Reichthum an Bindegewebe. Es werden hier auch sehr reichlich Gefässe angetroffen, einige mit grossen Lumina und starker Muscu- 
354 Katz, Ueber Blutanhäufungen bei doppelten Genitalien etc.

laris. - Kleinzellige Infiltrationen, die auf einen entzündlichen Zustand der Musculatur deuten könnten, sind nirgends vorhanden:

An der Verbindungsstelle mit dem Uterus wurden mehrfach Schnitte durch die bindegewebigen und musculären Verbindungsstränge gelegt, nirgends fand sich ein Lumen, und nirgends kleinzellige Infiltration.

Bezüglich der Structur der Tube, von der ich an den verschiedensten Stellen Schnitte angefertigt habe, wollen wir unterscheiden:

1. den histologischen Bau des ampullären Theils nach dem uterinen Ende zu

2. den Uebergang des Mittelstückes in die eigentliche Haematosalpinx und

3. den Haematosalpinxsack selbst.

In 1 sehen wir die Schleimhautfalten zum grossen Theil noch erhalten, die Epithelzellen der Schleimhaut sind, wenn auch nicht überall, so doch an fast allen Stellen vorhanden, jedoch zeigen - dieselben nirgends die Form von Cylinderzellen, sondern sind sehr abgeplattet und ziemlich niedrig. Die Schleimhautfalten enthalten wenige, allerdings sehr erweiterte Gefässe und vor allem an vielen Stellen hämorhagische Herde.

Die Musculatur ist stark hypertrophisch und ist an vielen Stellen durch Blutaustritt auseinander gedrängt. Ferner sind eine Menge grosser Gefässe, die voll mit Blut anrefüllt sind, zu sehen. In der Umgebung der Gefässe sind hier namentlich in der Nähe des Peritonealüberzuges kleinere und grössere Rundzellenanhäufungen.

In 2 hat die Zabl und die Grösse der Schleimhautfalten bereits bedeutend abgenommen. Die hämorrhagischen Herde in den Falten sind bedeutend grösser und vom Epithel der Schleimhaut ist nur noch wenig zu sehen. Nur an vereinzelten Stellen sind ganz platte, spindelförmige Zellen vorhanden. - Die Muscularis enthält eine sehr grosse Menge von stark hypertrophischen Gefässen, die mit Blut voligepfropft sind, und zahılreiche hämorrhagische Herde. - Die Muscularis ist dicker als bei 1, jedoch nicht infolge der Vermehrung der Muskelelemente, sondern durch Vermehrung der Bindegewebselemente, - Subperitoneal trift man vereinzelt kleinzellige Infiltrationen an.

Was endlich die Wand des eigentlichen Hämatosalpinxsackes betrifft, so ist dieselbe erheblich düner als die des nicht erweiterten Tubentheils. 
Von der Schleimhaut ist hier so gut wie nichts übrig geblieben; die ganze eigentliche Mucosa ist durch das Hämatom zerstört, dessen Ueberreste sich als eine gleichmässige, krümelige Masse darstellen, von denen nur noch hier und da ein Blutkörperchen zu erkennen ist. Inmitten dieser ungeformten Massen finden sich an verschiedenen Stellen Anhäufungen von Zellen mit deutlichem Kerne, die eine bestimmte Anordnung zeigen und noch einen deutlichen epithelialen Charakter erkennen lassen. Hier und da sieht man eine dünne Bindegewebslamelle, auf der die Zellen aufsitzen. Es sind diese Stellen wahrscheinlich als abgesprengte, in die Blutmassen hineingetriebene Schleimhautfalten anzusprechen.

Die Musculatur zeigt hier ebenfalls eine starke Hypertrophie, hier jedoch bedingt durch eine Vermehrung der musculären Elemente. Ausserdem befinden sich hier zahlreiche Pigmentschollen von braunrother Farbe, die dureh die ganze Musculatur ziehen. Das Pigment stellt sich in Form grösserer und kleinerer polymorpher Schollen und Körner dar. Gefässe sind nur in geringer Zahl vorhanden. Im intermusculären Bindegewebe finden sich vereinzelte rundzellige Infiltrationen.

Auch das Perisalpingium schliesst an einzellen Stellen rothbraune Pigmentkörnchen ein.

Das Ovarium wurde ebenfalls mikroskopisch uniersucht, der Befund bietet jedoch nichts Erwähnenswerthes.

Fassen wir die vorliegenden anatomischen Veränderungen zusammen, so haben wir eine Hämatometra im atretischen Nebenhorn eines Uterus bicornis unicollis mit rechtsseitiger Hämatosalpinx vor uns.

Bekanntlich wird der Genitaltractus so gebildet, dass die beiden Müller'schen Gänge sich zuerst aneinanderlegen, um dann vom Ende des 2. Embryonalmonats an theilweise zu verschmelzen. Dies geschieht in der Weise, dass das Septum verschwindet, das untere Drittel zur Scheide und das mittlere zum Uterus wird. Oben bleiben sie getrennt und bilden die Tuben. Verschmilzt nun der unterste Theil zar Scheide, der mittlere jedoch nur theilweise, und zwar nur in seinem unteren Theil (Cervicaltheil), so entsteht ein Uterus bicornis unicollis. Blejbt der eine der Gänge oberhalb der Scheidenpartie im Wachsthum zurück oder ist er bereits ursprünglich rudimentär angelegt, so wird dieser Thejl des Uterus weniger entwickelt: es entsteht ein sogenanntes rudimentäres Uterushorn. Je nach dem Grade der Hemmungsbildung kann selbstverständlich 
die Grösse und Form dieses verkümmerten Horns variiren. Bei den höchsten Graden der Verkümmerung ist das eine Horn nur als ein bandartiger, dünner (musculöser) Streifen oder als rundlicher Strang ohne Höhle vorhanden, der dann zumeist in der Gegend des inneren Muttermundes von dem ausgebildeten Horne seitlich abgeht. - Bei Verkümmerungen geringeren Grades stellt das betreffende Horn einen ausgehöhlten musculösen Strang dar, welcher in einen Eileiter ausläuft, Ovarium und Lig. ovarii proprium sind vorhanden. Den Anfang des Eileiters erkennt man an dem Abgange des Ligamentum latum. - Das Verbindungsstück mit dem entwickelten Horn ist bald solide, musculös, wie in unserem Falle, wo bindegewebige, musculöse Stränge vom Fundus bis zum inneren Muttermund, zwischen dem normalen Uterus und dem atretischen Horn verliefen, bald hohl, so dass dann eine offene Verbindung zwischen den beiden Hörnern besteht.

Nach Hegar (13) verläuft in dem Verbindungsstücke zu dem Uterushorne eine Arterie, welche als Art. uterina dieser Seite aufzufassen ist. - Ich habe bei der mikroskopischen Untersuchung des Verbindungsstückes ebenfalls grosse Gefässdurchschnitte gefunden.

Ist nun die Höhle des Nebenhorns mit functionsfähiger Schleimhaut ausgekleidet, so kann auch in diesem Horne eine Menstruation stattfinden, und zwar kann dieselbe ohne die geringste Störung erfolgen, falls das Nebenhorn in offener Verbindung mit dem Uterus. steht. Bei Verschluss des Verbindungsstückes kommt es zur Ansammlung von Menstrualblut in dem Nebenhorn, es bildet sich eine Hämatometra.

Typische Formen von Hämatometra im Nebenhorn eines Uterus bicornis unicollis sind nicht gerade sehr häufig in der Literatur. Es hat sich die Vermuthung Hegar's, der 1874 die beiden ersten derartigen Fälle beschrieb, dass eine Hämatometrabildung in einem rudimentären Uterushorn kein so seltenes Ereigniss sei, keineswegs bewahrheitet.

Die gewöhnliche Complication bei einem hohlen, mit Schleimhaut ausgekleideten Uterushorn ist die mit Schwangerschaft. In jüngster Zeit hat Kehrer (16) 12 Fälle von Hämatometra im Nebenhorn zusammengestellt gegenüber 36 Beobachtungen von Sehwangerschaft. Es ist lebhaft darüber discutirt worden, ob Hämatometra und Schwangerschaft in einem rudimentären Uterus- 
horn nebeneinander bestehen können. Werth (54) und bald danach Sänger (43) und Landau (22) betonten energisch, dass sich eine Gravidität und eine vorher bestehende Hämatometra in einem rudimentären Horne gegenseitig fast völlig ausschliessen, oder, um mit L. Landau zu reden, „das Nebenhorn des Uterus muss amenorrhoisch sein, damit es geschwängert werden kann".

Hieraus geht die sehr interessante Thatsache hervor, die bereits Gebhard (11) erwähnt, dass die Uterusschleimhaut, um zur Bergung und Einnistung des Eies befähigt zu sein, keineswegs der Menstruation bedarf. Man nahm früher nämlich allgemein an, dass die Menstruation ein Process sei, der die Uterusschleimhaut zur Bergung des Eies gewissermaassen vorbereitet.

Ich habe Fälle von Hämatometra im Nebenhorn eines Uterus bicornis unicollis im Ganzen 16 in der Literatur, mit meinem also 17, gefunden.

Im Folgenden will ich die Fälle aus der Literatur zusammenstellen, die mit meinem oben beschriebenen Fall übereinstimmen, also nur solche Fälle, wo eine Hämatometra im Nebenhorn eines Uterus bicornis unicollis bestand. Auf die Fälle von Hämatometra im Horn eines Uterus bilocuiaris, wie sie Werth (54), Zillessen (57) und in jüngster Zeit Opitz (33) beschrieben, will ich nicht ausführlicher eingehen, obgleich sie den von mir zu beschreibenden sehr ähnlich sind. Ich entnehme die zusammengestellten Fälle zum Theil aus den Arbeiten von Boije (2) und Kehrer (16).

Fall 1 wurde von Stoltz und Lobstein (47) beschrieben. Der Fall scheint bei Lebzeiten nicht beobachtet worden zu sein, sondern wurde 1821 von den beiden genannten Autoren in Strassburg bei einer Section gefunden. Livius Fürst (8) referirt ihn mit folgenden Worten: $n-$ - vom unteren Theile des linken Randes dieses Hornes ging ein solides Rudiment des linken Hornes in Gestalt eines fibrösen Stranges, das in einem fleischigen, taubeneigrossen Kolben endete. In ihm fand sich eine Höhle von der Grösse einer Mandel, die einen Blutpfropf enthielt."

Die beiden nächsten Fälle dieser Art wurden von Hegar (13) beobachtet:

Fall 2, 26jähriges Mädchen, das seit dem 14. Jahre regelmässig ohne Schmerzen menstruirt war, im 20. Jahre längere Zeit Krampfanfälle im Unterleib; mit 25 Jahren erfolgte 14 Tage nach Beendigung 
der letzten Periode im Anschluss an eine Ueberanstrengung eine Blutung. Danach wurden die Menses reichlicher, anteponirend und schmerzhaft, später spärlicher und postponirend. Mit Erscheinungen von Peritonitis und zunebmender Abmagerung trat die Pat. in die Freiburger Klinik ein. Der linke, normal grosse Uteruskörper lag in Retroversión und nach der linken Seite geneigt; an das Collum legte sich nacb rechts hin ein gänseeigrosser, schmerzhafter Tumor an, der das Scheidengewölbe nicht herabdrängte, aber auf ihm ruhte. Per rectum fühlte Hegar einen bleifederdicken, $3 \mathrm{~cm}$ langen Strang zwischen Cervix des linken Horns und dem Tumor; er stellte die richtige Diagnose, für die er eine sichere Stütze durch die Probepunction. durch die Bauchdecken gewann, die eine dicke theerähnliche Blutmasse ergab. Der Rest des Inhalts wurde später mittels Punction durch das rechte Scheidengewölbe hindurch entleert.

Fall 3. 19jähriges Mädchen, das seit 4 Jahren menstruirt war. Erste Periode schmerzlos; die folgenden unregelmässig, 7 Tage dauernd, mit starken Schmerzen verbunden. Im Intervall selbst häufige Schmerzen im rechten Hypogastrium. - Nach rechts und hinten von der normal stehenden Portio lag eine apfelgrosse, druckempfindliche Geschwulst, die das hintere Scheidengewölbe rechts stark herabdrängte. Zwischen dem nach links geneigten Uterushorn und dem Tumor bestand eine Furche, in deren Tiefe ein Verbindungsstrang beider wahrgenommen wurde. - Durch das Scheidengewölbe hindurch wurde eine Probepunction gemacht, bauptsächlich um über die Dicke der Wand Aufschluss zu erhalten; dieselbe wurde auf $1 \frac{1}{2} \mathrm{~cm}$ geschätzt. Der Inhalt des Tumors bestand aus Blut.

Fall 4 wurde von Frankenhäuser beobachtet, von H. Meyer (29) in seiner Dissertation beschrieben.

Eine 26jährige Magd war im 15. Jahre zuerst und von da $a b$ regelmässig alle 4 Wochen menstruirt; meist dauerte der blutige Ausfluss einen bis zwei Tage mässig stark an, pflegte dann einen Tag fast ganz auszusetzen, danach einen bis zwei Tage profus zu werden; am zweiten und dritten Tage, zur Zeit, da die Blutung fehlte, Schmerzen, die mit beginnender Blutung aufhörten. Das Blut war zum Theil zu schwarzen, kleinen Klümpchen geronnen. Mit der Portio stand ein sinistroponirtes, anteflectirtes, spitz zulaufendes Uterushorn in Verbindung, von dessen rechter Seite viel tiefer als am Fundus ein breiter Stiel zu einem hühnereigrossen, elastischen Tumor führte, der mit dicken Wandungen versehen war. Bei Druck auf die Blutgeschwulst entleerte sich unter Klein- und Schlaffwerden des Tumors etwas schwarzes Blut aus dem dem linken Horn angehörenden Os externum. Trotz dieser, wenn auch engen, Communication zwischen Blutsack und der Cervix des linken Horns gelang es nicht, die Sonde in den Tumor einzuführen.

Die Pat. wurde keiner Behandlung unterworfen, sondern unter Beobachtung gestellt.

Fall 5. Von Leopold (23) beobachtet, der insofern grösseres Interesse verdient, als $>$ er der erste ist, bei dem eine anatomische Diagnose gemacht werden konnte. 
Ein 24jähriges Fräulein, seit dem 15. Jahre menstruirt, litt seit über 2 Jahren während der Periode an heftigen Schmerzen. Nach einer blutigen Erweiterung des stenosirten Cervixcanals trat Gravidität ein. Nach der Geburt des Kindes viel stärkere Schmerzen zur Zeit der Periode. Links von der Medianlinie des Körpers lag das sondirbare linke Uterushorn; ron seinem Os internum etwa ging nach rechts ein Strang $a b$, der zu einem apfelgrossen, harten Körper fübrte. Probelaparotomie auf Grund der richtigen Diagnose ohne weiteren Eingriff. Von der zweitnächsten Periode an traten die früheren Schmerzen, immer heftiger werdend, wieder auf; bei jeder Menstruation schwoll das rechte Uterushorn zu einer äusserst druckempfindlichen Kugel an, von deren oberem rechten Umfange sich die Tube als ein wurstartiger Strang abhob. Bei der zweiten Laparotomie wurde die Salpingo-oophorectomia duplex zum Zwecke der Verhinderung weiteren menstruellen Blutandranges zum atretischen Nebenhorn vorgenommen. Dauernde Heilung, obwohl das blatgefüllte 'Nebenhorn zurückblieb. Sein Inhalt wurde später resorbirt. - $\mathrm{Zu}$ bemerken ist noch, dass sich inzwischen eine leichte rechtsseitige Hämatosalpinx gebildet hatte, die eine weitere Indication für die zweite Operation abgab.

berichtet.

Fall 6 ist von Czerny operirt und von E. v. Meyer (28)

Die Pat., 21 Jahre alt, ledig; bekam im 14. Jahre die Regel; schon von Anfang an dysmenorrhoische Beschwerden, indem einige Tage vor dem Termin schmerzhafte Anschwellung des Leibes und darauf 2 bis 3 Tage dauernde Blutung mit heftigen Schmerzen im Kreuze und rechten Oberschenkel auftraten. Seit 8 Wochen in der rechten Bauchseite ein drückendes Gefühl. Die Untersuchung ergiebt: Uterus von $6 \mathrm{~cm}$ innerer Länge, mit Corpus antevertirt und nach links gelagert. Am rechten hinteren Scheidengewölbe ein enteneigrosser, weich elastischer Körper, der sich nach oben bisquitförmig zu einer zweiten kugeligen Erweiterung fortsetzt: Nach innen davor ein kugelförmiger Körper, der im vorderen Scheidengewölbe zu fühlen ist. - Es wurde Salpingo-oophorectomia duplex abdominal ausgeführt.

Die anatomische Diagnose lautete: Uterus bicornis unicollis, Haematometra dextra, Haematosalpinx dextra. Cystische Entartung des Ovariums. Links: Katarrhalische Schwellung der Tube und kleincystische Degeneration des Ovariums. - Heilung.

Fall 7. Citirt von Kehrer.

Salkowski-Warschau (45) berichtet 1894 über einen Fall von Blutansammlung im rudimentären Nebenhorn und der Tube eines Uterus bicornis unicollis, der dureh Laparotomie geheilt wurde.

Fall 8 wurde beobachtet ron Mangiagalli (27). Die Beschreibung desselben ist jedoch etwas unvollständig.

Die Menses begannen bei der 18jährigen Patientin mit 14 Jahren und waren dann regelmässig gewesen. Seit einem Jahre Schmerzen im Abdomen. Die letzten drei Ilenses waren weniger blutreich als vorher. 
Die äusseren Genitalien gesund, sicher virginell. Uterus klein, beweglich, anteflectirt, etwas nach links gezogen. Durch das rechte Scheidengewölbe ein runder, eigrosser Tumor, deutlich durch eine Furche von der Gebärmutter abgegrenzt, sehr druckempfindlich und unten im Becken fixirt. Bei der Laparotomie findet man einen Uterus mit wohl entwickeltem linken Horn, sich in die normal gebildete Portio vaginalis fortsetzend, und ein etwa dreimal so grosses, bypertrophisches rechtes Horn von sehr fester Consistenz. Dieses Horn wird atretisch gefunden. In der Vermuthung, dass dieses Horn retinirtes Menstrualblut entbält, wird es exstirpirt. Die Wand sehr dick. "La carita era tappezzata da uno strato di sanguo coagulato dello spessore di qualche millimetro." - Ueber den Ausgang der Operation und die Heilung ist nichts berichtet.

Fall 9. Sänger (43) beschreibt folgenden tall von Haematometra cornu sinistri uteri bicornis:

Eine seit $2^{1 / 2}$ Jahren verbeirathete, 29 jährige Nullipara hatte seit 11-12 Jahren als Andeutung der Menstruation in Zwischenräumen von etwa 24 Tagen Abgang von ganz wenigen Tropfen Bluts unter heftigen krampfartigen Schmerzen in der linken Seite des Unterleibs. Dem nach rechts geneigten Corpus uteri sass links ein hühnereigrosser, unempfindlicher, praller Tumor ohne tastbaren Verbindungstrang auf, der Hämatometrasack. - Auch das rechte offene Horn, mit abnorm kurzer Tube, sehr kümmerlich entwickelt.

Abdom. Salpingo-oophorectomia duplex. Heilung. (Die Haematometra war hühnereigross.)

Fall 10, von E. Rossa (40) beschrieben.

29jährige Frau; mit 14 Jahren menstruirt, mit dem 17. Jahre Schmerzen während der Periode, die im letzten Jahre besonders heftig waren. Eine Frühgeburt von 7 Monaten. Im Abdomen ein bis zum Nabel reichender, nach links gelagerter Tumor; durch eine Furche von ihm getrennt der normal grosse, anteflectirte, nach rechts gedrängte Uterus. Bei der Laparotomie ergab die Punction des grossen Sackes eine dickflüssige chokoladebreiähnliche Masse.

Von der Gegend des Orific. int. ging nach links ein solider, $1 \mathrm{~cm}$ dicker, runder Strang ab, der nach $2 \mathrm{~cm}$ in einen $8 \mathrm{~cm}$ Iangen, ovalen, an der breitesten Stelle $6 \mathrm{~cm}$ breiten Körper überging; er adhärirte in Folge peritonitischer Verwachsungen der vorderen Fläche des tief in's kleiue Becken reichenden Tumors. Vom oberen Ende dieses atretischen linken Nebenhorns entsprang die linke Tube; aber nur im Anfang ihres Verlaufes war sie normal beschaffen, sehr bald ging sie in die grosse Hämatosalpinx über, deren Wände zum Theil nekrotisch waren. Das linke Ovarium fehlte. - Das atretische Uterushorn sammt Adnexen wurde per laparotomiam entfernt. -- Heilung.

Im linken Uterushorn fand sich eine nach unten abgeschlossene, $5 \mathrm{em}$ lange und $3 \mathrm{~cm}$ breite, mit theerartiger Masse ausgefüllte Höhle. In der linken Tubengeschwalst eine dickfiussige, chokoladefarbige Masse.

Fall 11, beschrieben von Calmann aus dem Institut von Rosthorn in Prag.

22 jährige Jedige Person, war seit dem 17. Jahre stets unregelmässig, in 6-8 wöchentlichen Pauseu, reichlich und sehr schmerzhaft 
menstruirt. Mit 19 Jahren einige Tage heftige Schmerzen im Unterleib. Seit 2-3 Monaten bemerkt sie das allmähliche Wachsen einer Geschwulst in der rechten Unterbauchgegend.

Linkes Uterushorn atrophisch, retrovertirt und nach links gedrängt; rechts neben ihm und durch einen kleinfingerdicken Strang mit ihm verbunden ein bis an die Beckenwand reichender, faustgrosser Tumor: das durch menstruelles Blut ausgedehnte rechte Nebenhorn. Laparotomie; zunächst Fixation des Bauchsacks an die Bauchdecken, dann Abtragen von Ovarium und Tube der rechten Seite. Da sich 2 Monate später wiederum eine Menstruation mit sehr lebhaften, unaufbörlichen Schmerzen einstellte, entschloss sich Rosthorn zur zweiten Laparotomie, bei der das dickwandige, mit zähem, chokoladefarbenem Blute gefüllte Nebenhorn trotz seiner Adhäsionen mit der Nachbarschaft exstirpirt wurde. Es zeigte Sanduhrform; die Einschnürung entsprach der Gegend des Os internum. Nikroskopisch fand Calmann in der oberen Hälfte des Blatsacks eine normale Schleimhaut, die trotz des hohen, im Sack herrschenden Drucks nicht abgeplattet, sondern an zahlreichen Stellen gut ausgebildet wal: in den dem Collum entsprechenden Wandtheilen wurde das Cylinderepithel aber höher, die Drüsen und Gefässe zahlreicher und auf einer Schleimhautfalte war statt der Cylinderepithelien Plattenepithel, das in Form solider Zapfen atypisch in die Tiefe wucherte. - Eine Communication mit dem entwickelten Horn war nicht zu entdecken.

\section{Fall 12. Im Jahre 1898 demonstrirte Winter (56) in der} Gesellschaft für Geburtshülfe und Gynäkologie in Berlin folgenden Fall :

"Die Kranke hatte mit 16 Jahren die erste Menstruation bekommen und erst mit 19 Jahren hatten bei ihr die ersten Dysmenorrhoen begonnen und sich im Laufe von 4 Jahren immer heftiger gestaltet." Es bestand eine Hämatumetra im rudimentären Horn eines Uterus bicornis bei einfacher Cervix und einfacher Vagina. Die Exstirpation des rudimentären Horns wird ohne Schwierigkeit per laparotomiam ausgeführt. "Das Horn ist apfelgross und zeigt an der Abtragungsstelle eine $1 \mathrm{~cm}$ dicke Muskulatur, während sie am entgegengesetzten Ende in der Nähe des Tubenostiums nur einige Millimeter dick ist. Die Höhle enthielt höchstens 2 Esslöffel schwarzen dünnflüssigen Blutes."

\section{Fall 13 beschreibt Boije (2).}

Bei der 27jährigen Pat. hatten sich die Menses mit 14 Jahren eingestellt. Dieselben waren stets regelmässig mit 3 wöchentlichen Intervallen und 6-7 tägiger Dauer wiedergekehrt und immer mit Schmerzen verbunden gewesen, die allmählich gegen Ende der Menstruation zunahmen. ln den Zwischenpausen füblte sicb Pat. völlig gesund. Sie heirathete mit 22 Jahren und hat 3 Partus mit ungestörten Puerperien durchgemacht.

In der letzten Zeit wurden die Schmerzen bei den Menses immer stärker, dauerten bald auch in den Intervallen fort und waren zeitweise so stark und mit einem Gefühl von Krampf rechts unten im Unterleib verbunden, dass die Pat. meist zu Bett liegen musste. - Uterus anteflectirt, klein, mit der Cervix leicht nach links gezogen. Portio vagi- 
nalis im stumpfen Winkel zur Scheideuachse. Rechts vom Uterus ein hühnereigrosser, abgerundeter, beweglicher, empfindlicher Tumor von fester Consistenz, der mit einem ebenfalls festen, cylindrischen Stiel von der Dicke einer Bleifeder vom rechten Uterushorn ausgeht. - Ausserhalb dieses Tumors tief unten in der rechten Seite der Excavation wird ein anderer kleinerer Körper palpirt (rechtes Ovarium?).

Diagnose: Enaometritis. Myoma uteri. Perioophoritis sinistra. Residuae Parametritis sinistrae. - Die Laparotomie ergiebt ein rudimentäres rechtes Uterushorn, von dessen äusserem aberen Umfang eine kurze atrophische Tube abgeht. Der Tumor hat ungefähr die Form eines Fis und ist mit der rechten Seite des ausgebildeten Uterus durch einen $1 \frac{1}{2} \mathrm{~cm}$ langen, fast cylindrischen, doch von vorn nach hinten zu etwas abgeplatteten, an der Basis etwas dickeren Stiel verbunden. Exstirpation des Horns, nach Unterbindung mit Catgut. - Communication des Tumors mit der Tube oder der Uterinhöhle lässt sich makroskopisch nicht nachweisen. Bei Eröffnung des Tumors enthält derselbe etwas über 2 Theelöffel dunkelfarbigen, dickflüssigen Blutes, welches herausgepresst wird, während sich die Wand zusammenzieht. Dieselbe ist überall etwa $1 \frac{1}{2} \mathrm{~cm}$ dick. - Die innere Fläche des Tumors von hellbraunem, sammetartigem, schleimhautähnlichem Aussehen.

Heilurgsverlauf ungestört. Die Operirte wurde am 15. Tage als gesund entlassen. Die Menses sind nach der Operation regelmässig mit 3 wöchentlichen Intervallen und nur 3 tägiger Dauer wiedergekehrt und völlig schmerzlos gewesen. 'Die Kräfte nahmen von Tag zu Tag zu und die Pat. fühlte sich völlig gesund.

Die mikroskopische Untersuchung ergiebt folgenden Befund: Die Wandung der Höhle innen überall mit Schleimhaut bekleidet, welche der Hauptsache nach gewöhnlicher Uterusschleimbaut gleicht. Oberfläche mit Cylinderepithel bekleidet; dieses ist an den meisten Stellen von normalem Aussehen aus cylindrischen Zellen bestehend. An andern Stellen sind die Zellen mehr kubisch. - Das interglanduläre Gewebe zeigt kleinzellige Infiltration, die Drüsen sind an einigen Stellen sebr reichlich und zeigen dasselbe Bild wie bei der hyperplastischen Endometritisform.

In der Musculatur fällt der grosse Gefässreichthum auf. Im Stiel des exstirpirten Horns ist auch bei der mikroskopischen Untersuchung kein mit Schleimhaut bekleideter Canal zu finden.

\section{Fall 14. Halban (12) beschreibt folgenden Fall:}

Bei der 17 jährigen Patientin stellten sich mit dem Auftreten der Menstruation heftige Kopfschmerzen, Bauchschmerzen, zeitweilig auch Erbrechen ein, ein Zustand, welcher die 8 Tage dauernde Menstruation stets um einige Tage noch überdauerte. Menstruation anfangs unregelmässig, blieb mehrere Monate aus, später wurde sie regelmässig, 4 wöchentlich, 3-4 tügig, reichlich. - Seit mehreren Jahren bemerkte Pat. eine harte Geschwulst in der rechten Unterbauchgegend, welche zur Zeit der-Menstruation grösser wurde. Patientin war niemals gravide. Uterus in Anteversio-flexio, rechts vom Uterus ein hïhnereigrosser, gestielter Tumor von glatter Oberfläche, harter Consistenz, gut beweglich, etwas druckempfindlich. Von diesem Tumor aus zieht zur rechten seitlichen Uteruswand ein ca. bleistiftdicker Strang, von der unteren Fläche des Tumors zieht wieder ein Strang gegen den rechten Leisten- 
canal, derselbe ist bimanuell deutlich zu tasten, über bleistiftdick, etwas druckempfindlich. Dieser Strang geht im Leistencanal in eine knotige, über haselnussgrose Auftreibung über. -

Ovarien beiderseits zu tasten, das linke Ovarium auf das 3 fache vergrôssert, descendirt.

Diagnose: Tumoren des Lig. rotundum, von denen der eine intraperitoneal, der andere zum Theil im Leistencanale, zum Theil ausserhalb desselben gelagert war. Laparotomie. Suprasympbysärer Kreuzschnitt (Küstner). Exstirpation des rudimentaren Horns. Durchtrennung des Stranges, welcher vom rudimentären Uterushorn in den Leistencanal zieht (Lig. rotundum). - Rechtes Ovarium normal. -Linkes Ovarium über nussgross, die mediale Hälfte, bläulich durchscheinend, wird incidirt, wobei sich altes, dickftüssiges Blut in einer Menge von ungefähr $3 \mathrm{ccm}$ entleert. Vernähung der Incisionswunde. - Freipräparirung des in rechtsseitigen Inguinalcanal gelegenen Tumors. Vollständig normale Reconvalescenz. Pat. verlässt am 14. Tage das Bett.

Der exstirpirte Tumor (rudimentäres Nebenhorn) wird unmittelbar nach der Operatiou aufgeschnitten. Es findet sieh in ihm eine ungefähr fingerhutgrosse, mit Schleimhaut ausgekleidete Höhle, welche mit altem dicklichen Blute ausgefüllt ist. Die Wand des Nebenhorns selbst ist stark hypertrophirt, in ihr einzelne myomatöse Knoten.

Die mikroskopische Untersuchung ergiebt normale Drüsen, das Epithel der Oberflächenschleiminaut selbst an vielen Stellen fehlend. Die Myomknoten erweisen sich als Adenomyome (Recklinghausen).

Der Verbindungsstrang $z$ wischen entwickeltem und rudimentärem Uterushorn enthält keinen Canal.

\section{Fall 15, beschrieben von A. Pinna Pintor (Turin) (36).}

Bei der 18jährigen Pat, war die Periode während der ersten drei Jahre völlig normal. Seit dem 18. Jahr Schmerzen bei der Menstruation. Dieselben verschwanden mit dem Beginn der ersten Schwangerschaft, und auch in der Zwischenzeit $z$ wischen dieser und den folgenden Schwangerschaften blieben sie aus; erst 2 Jahre nach der letzten Entbindung traten die Schmerzen von neuem auf, wenn auch (im Anfange wenigstens) nicht bei jeder Perjode. Kein Fluor albus. Die Untersuchung ergab rechts neben dem Uterus - und von diesem nur durch eine leichte sagittale Furche getrennt - einen rundlichen, ziemlich harten, hübnereigrossen Tumor; Länge der Uterushöhle normal, ebenso die Portio vaginalis. Der Tumor war mässig drackempfindlich. Die Diagnose schwankte awischen Adnextumor und Fibromyom.

Der operative Eingriff wurde mittelst vorderer Kolpotomie (Kreuzschnitt) vorgenommen. Der Tumor zeigte zahlreiche Adhäsionen mit der Umgebung; derselbe bildete mit dem eigentlichen Uteruskörper ein untrennbares Ganze, und enthielt (wie eine Probeincision während der Operation ergab) eine mässige Menge pechfarbenen, flüssigen Blutes. Die genauere Untersuchung bewies, dass es sich in der That um eine Blutretention im rudimentären rechten Uterushorn handelte. Die rechtsseitigen Adnexe sind degenerirt und atrophisch, die linksseitigen normal. Bei der breitbasigen Verbindung der beiden Uterushälften war eine Resektion schwer ausführbai"; es wurde daher die Totalexstirpation mit Zurücklassen der linken Adnexe - vorgenommen. Glatter Heilungsverlauf. 
$364 \mathrm{Katz}$, Ueber Blutanhä̈ufungen bei doppelten Genitalien etc.

Tabelle über die Fälle von Hämatometra im

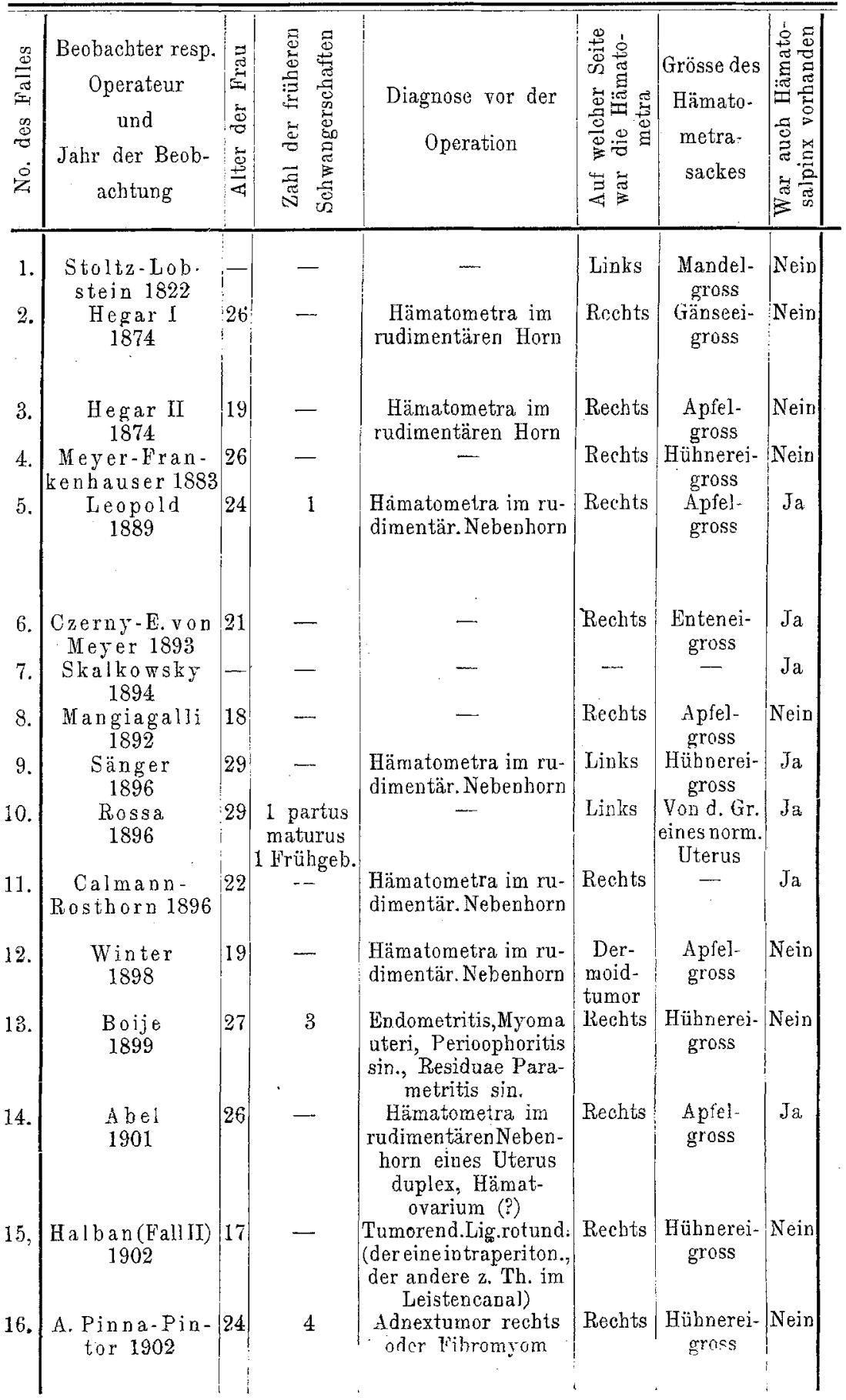


Nebenhorn eines Uterus bicornis unicollis.

\begin{tabular}{|c|c|c|}
\hline Therapie & Erfolg der Operation & B emerkungen \\
\hline - & - & Bei der Section ge- \\
\hline $\begin{array}{l}\text { Application ron Kali causticum } \\
\text { - Stift seitlich von der } \\
\text { Portio - Als diese ohne } \\
\text { Erfolg, } 2 \text { Mon. später Punction }\end{array}$ & $\begin{array}{l}\text { Entstehung einer Blasen- } \\
\text { scheidenfistel und Blasen- } \\
\text { Nebenhornfistel }\end{array}$ & - \\
\hline Punction & Heilung & - \\
\hline $\begin{array}{l}\text { Entleerung des Sacks durch } \\
\text { Compression desselben }\end{array}$ & Heilung & $\begin{array}{l}\text { Verbindungstrang } \\
\text { offenb.engeanalisirt }\end{array}$ \\
\hline $\begin{array}{l}\text { Erste Probelaparotomie, später } \\
\text { wegen stärkerer Beschwerden } \\
\text { Salpingo - oophorectomia du- } \\
\text { plex. Hämatometra blieb } \\
\text { zurück. }\end{array}$ & Heilung & - \\
\hline Salpingo-oophorectomia duplex & Heilung & - \\
\hline Laparotomie & Heilung & - \\
\hline $\begin{array}{l}\text { Exstirpation des Horns per } \\
\text { laparotomiam }\end{array}$ & Nichts vermerkt & - \\
\hline Salpingo-0ophorectomia duplex & Heilung & - \\
\hline $\begin{array}{l}\text { Punction der Hämatosalpinx, } \\
\text { dann Exstirpation der Hä- } \\
\text { matometra u. Hämatosalpinx }\end{array}$ & Heilung & $\begin{array}{l}\text { Beid. Blutsäckedurch } \\
\text { peritonitische Mem- } \\
\text { branen verwachsen }\end{array}$ \\
\hline $\begin{array}{l}\text { Erst Salpingo-oophorectomia } \\
\text { dextra, bei der } 2 \text {. Laparot. } \\
\text { Exstirpation d. Hämatometra }\end{array}$ & Heilung & - \\
\hline $\begin{array}{l}\text { Exstirpation des rudimentären } \\
\text { Horns per laparotomiam }\end{array}$ & Heilung & - \\
\hline $\begin{array}{l}\text { Exstirpation des rudimentären } \\
\text { Horns per laparotomiam }\end{array}$ & Heilung & - \\
\hline $\begin{array}{l}\text { Vaginale Exstirpation des } \\
\text { rudimentären Horns und der } \\
\text { rechten Adnexe }\end{array}$ & Heilung & -- \\
\hline $\begin{array}{l}\text { Exstirpation des rudimentären } \\
\text { Nebenhorns (suprasymphy- } \\
\text { särer Kreuzschnitt) }\end{array}$ & Heilung & $\begin{array}{l}\text { Verbindungstrang } z w . \\
\text { entwickeltem u. ru- } \\
\text { diment. Uterushorn } \\
\text { enthältkeinen Camal }\end{array}$ \\
\hline $\begin{array}{l}\text { Vaginale Exstirpation des } \\
\text { ganzen Uterus nebst den } \\
\text { rechten Adnexen }\end{array}$ & Heilung & - \\
\hline
\end{tabular}


366 Katz, Ueber Blutanhäufungen bei doppelten Genitalien ete.

Die angeführten 16 Fälle von Hämatometra im atretischen Nebenhorn eines Uterus bicornis unicollis sind die wenigen, die ich in der Literatur gefunden. Fälle von Uterus bicornis mit rudimentärem Nebenhorn sind ziemlich häufig, auch solche mit einer von Schleimhaut ausgekleideten Höhle. Dass aber das rudimentäre Nebenhorn sich an der Menstruation betheiligt, kommt nicht gerade sehr oft vor. - Es sind aber auch, wie wir gesehen haben, zum Zustandekommen einer Haematometra eine ganze Menge Voraussetzungen nöthig. Vor allen Dingen ist es erforderlich, dass die Schleimhaut, welche die Höhle des Horns auskleidet, im Stande ist, zu menstruiren. Genaue mikroskopische Untersuchungen des rudimentären Horns scheinen jedoch nicht oft gemacht worden zu sein. Ausführliche Angaben darüber finden wir nur in den Fällen von Calmann, Boije, Halban. - - Im ersteren dieser Fälle war die Mucosa gut entwickelt, das Oberflächenepithel nirgends abgeplattet; im Fall Boije finden wir ebenfalls eine gut entwickelte Schleimbaut, deren Oberflächenepithel überall intact ist, an einigen Stellen sind kubische Zellen vorhanden. Die Drüsen sind vermehrt.

In Fall Halban und in meinem Falle fehit an vielen Stellen das Oberflächenepithel, an den meisten Stellen ist es in meinem Falle vollständig abgeplattet; wir haben es also in den Fällen, in denen eine mikroskopische Untersuchung vorliegt, mit einer Schleimhaut zu thun, die die Bedingungen einer menstruellen Congestion erfüllen kann. Andererseits müssen wir anführen, dass so und so ott in Präparaten von Hämatometra bej rudimentärem Nebenhorn, die man bei der Section gefunden, und auch ron Werth und Ziilessen in ihren Fällen von Hämatometra im Uterus septus bilocularis - Fällen, die den unsrigen sehr ähnlich sind eine ungenügend ausgebildete Schleimhaut entdeckt wurde. Die Compression der Schleimhaut kann z. B. in den beiden letzterwähnten Fällen unmöglich diese Veränderungen herbeigeführt haben. Es müssen also noch andere Einflüsse sein, die hier eine Rolle spielen; welcher Art dieselben sind, ist bei dem Mangel an diesbezüglichen Untersuchungen, vorläufig unmöglich zu entșcheiden. Kehrer sagt: "Für die Mehrzahl der Fälle von eigentlichem rudimentären Nebenhorn ist anzunehmen, dass das besser entwickelte Horn die menstruelle Blutausscheidung zu besorgen pflegt, während das Nebenhorn - nicht unbedingt abhängig davon, ob seine Mucosa gut oder schlecht entwickelt ist - vollkommen amenorrhoisch oder wenigstens eine kaum bemerkbare Menge Blut in 
sein Cavum abscheidet. Vielleicht reagirt es nur mit einer menstruellen Schwellung."

In zweiter Linie ist zum Zustandekommen einer Hämatornetra im rudimentären Nebenhorn nothwendig, dass das Menstrualblut keinen freien Abfluss hat. Wir haben in unserem Fall eine breitbasige bindegewebig-muskulöse Verbindung mit dem Uterus, in dem auch mikroskopisch kein Lumen festzustellen ist. Es dürfte wohl als ziemlich sicher anzunehmen sein, dass hier die Atresie des Verbindungsstückes congenitalen Ursprungs war. Selbst Veit (51) giebt zu, dass die Atresien bei doppelten Genitalien congenital entstanden sind.

Was die feineren anatomischen Verhältnisse betrifft, so sind dieselben, wie schont erwähnt, nur in einigen Fällen untersueht worden. Das lag einfach daran, dass bisher die Präparate nicht zugänglich waren, da die Behandlung meistens darauf hinzielte, durch Wegnahme der Adnexe die Menstruation zum Stillstand zu bringen.

Die anatomischen Verhältnisse und der klinische Verlauf zeigen, dass das im rudimentären Uterushorn retinirte Menstrualblut unter sehr hohem Drucke steht. Unter solchen Umständen sollte man annehmen, dass man Zeichen einer ausgedehnten Druckatrophie der Schleimhaut findet. Das ist aber absolut nicht der. Fall. Im Gegentheil fanden wir in den meisten Fällen ziemlich wohlerhaltene Schleimhaut. Schon früher haben Th. Landau und Rheinstein (21) nachgewiesen, wie widerstandsfähig die Schleimhaut gegen einen derartigen inneren Druck ist. ${ }_{n}$ Bei Missbildungen der Genitalien, seien es nun Verschlussbildungen oder Doppelbildungen, ist die Schleimhaut in allen Abschnitten des Genitaltractus wie in der Norm angelegt; die Uterusschleimhaut geht bei Rückhaltung des Menstrualblutes schliesslich durch Druckatrophie zu Grunde; die Körperschleimhaut erhält sich dabei auffallend lange widerstandsfähig." Boije fand sogar in seinem Falle die Epithelzellen, die ibre normale Form und Anordnung beibehalten hatten, mit lebhaft flimmernden Cilien versehen.

Ir meinem Falle waren allerdings an keiner Stelle die Epithelzellen von normaler Form, sondern meistens kubisch abgeplattet, sie hatten jedoch ihre epitheliale Natur beibehalten, und nur an wenigen Stellen fanden sich, ebenso wie in Boije's Falle, proliferirende-Zellenmassen, die ohne bestimmte Grenze in das klein- 
zellig infiltrirte Gewebe übergingen. - Es ist dies wohl kaum anders als durch Drucknekrose zu erklären.

Engström (5) nahm an, dass das in dem Uterus zurückgehaltene Menstrualblut zum Theil wenigstens resorbirt wird, und erklärt sich dadurch den Umstand, dass die Mucosa relativ unbedeutend verändert ist, trotz des hohen Druckes, dem sie ausgesetzt war.

Wenn. auch die Beschaffenheit des Blutes in dem rudimentären Uterushorn (theerartige Eindickung) geeignet ist, die Anschauung Engström's zu stützen, so muss andererseits hervorgehoben werden, dass nur in dem Falle Calmann (4) Zeichen von Blutpigment im Schlejmhautgewebe gefunden wurden. (Calmann deutete es als Rest der menstruellen Blutung). Auch in meinem Falle konnte ich trotz genauster Untersuchungen daraufhin nichts davon entdecken.

In allen beschriebenen Fällen, auch in meinem, fand sich eine nicht unbeträchtliche Dicke der Muskulatur. Diese beruht auf einer Arbeitshypertrophie, das muskulöse Hohlorgan jst bestrebt, seinen Inhalt auszutreiben, es antwortet "auf den gleichsam als Fremdkörper wirkenden Reiz (Kehrer) der Blutmasse durch wehenartige Contractionen und diese führen zur "Hypertrophie der Muscularis." Der Hämatometrasack war niemals grösser als ein Gänseei, im Durchschnitt war er apfel- oder hühnereigross.

Der klinische Verlauf bei einer Hämatometrabildung im rudimentären Nebenhorn eines Uterus bicornis, aus dem das Menstrualblut keinen Abfluss hat, lässt sich leicht erklären. Die, so zu sagen, typisch auftretenden und sehr heftigen Schmerzen, welche das ganze Krankheitsbild beherrschen, können zur Diagnose sehr verwerthet werden und legen beim Ausschliessen einer anderen Erklärung den Gedanken an eine Bildungshemmung nahe. -

Mainzer (25) sagt: "Handelt es sich um einen einseitig atretischen Uterus bicornis bei einfacher Scheide, so kann die richtige Deutung des Zustandes zu den allerschwierigsten Aufgaben der Diagnostik gehören. In solchen Fällen kann es schon ein Verdienst sein, an die Möglichkeit einer Doppelbildung gedacht zu haben."

In Bezug auf unseren Fall ist zu bemerken, dass sowohl die Anamnese als auch der Symptomencomplex so ziemlich das als typisch Geltende darboten. Die Hauptklage der Patientin bezog 
sich auf Schmerzen im Unterleib, welche einige Tage vor der Periode einsetzten und diese begleiteten. - Es ist anzunehmen, dass diese typischen Schmerzen auf Dehnung der Höhle durch retinirtes Menstrualblut und auf einer Contraction der Wandmuskulatur beruhten. Nicht immer jedoch stellen sich diese Schmerzen bei congenitalen Gynatresien in der einen Hälfte eines doppelten Genitalkanals mit consecutiver Ansammlung von Menstrualblut gleich im Beginn der Pubertät ein, sondern nach Pick (35) passirt es relativ häufig, dass die Frauen erst am Ende des 2. oder am Anfang des 3. Lebensdecenniums wegen dieser Zustände bezw. ihrer Folgen in ärztliche Behandlung kommen." Der Grund hierfür ist offenbar der, dass die Menstruation in solchen atretischen, bäufig rudimentären Uterushälften nur spärlich ngleichsam rudimentär" (vergl. Sänger (43) S. 51) statt hat. Die Bildung grösserer Hämatometren ist hier äusserst selten."

Welche Schwierigkeiten die Diagnose der Fälle von Hämatometra des rudimentären Nebenhorns bietet, geht auch aus dem Umstande hervor, dass nur $7 \mathrm{mal}$ in den angeführten 16 Fällen die Diagnose gestellt wurde. - In unserem Falle war ausser den typischen Schmerzen, die am 2. Tage der Menstruation stets noch zunahmen, häufig ruckweise auftraten und in der intermenstruellen Zeit verschwanden, auch der übrige Befund ein derartiger, dass man über die Natur der Erkrankung kaum im Zweifel sein konnte. Der Befund des unmittelbar dem Uterus zur rechten Seite anliegenden zweiten Tumors brachten Herrn Dr. Abel gleich auf den Gedanken, dass es sich um einen Uterus duplex handelte, und zwar, da keine zweite Portio bestand und ebenso wenig keine sonstigen Anomalien oder Missbildungen bei der Patientin nachzuweisen waren, um eine Blutansammlung im verschlossenen Nebenhori.

Wenn man also erst an die Möglichkeit dieser Affection denkt, dürfte es, wie schon Hegar hervorgehoben hat, nnicht schwer oder wenigstens nicht unmöglich" sein, die richtige Diagnose zu stellen. Boije sagt: Hat man einen abgerundeten Tumor von ziemlich fester Consistenz, der mit einem Körper von der typischen Form und Lage eines Uterus unicornis in Verbindung steht, und die Anamnese an die Hand giebt, dass die charakteristischen Symptome bestehen, so kann die Diagnose kaum zweifelhaft sein. - Eine Verwechselung mit einem Myom ist das einzige, was in Betracht kommen könnte. Ist man im Zweife] hierüber, so hätte man in 
einer Probepunction ein geeignetes, wenn auch nicht ganz ungefährliches Mittel zur Sicherstellung der Diagnose, da bei einer Hämatometra der Inhalt von charakteristischem Aussehen ist.

Unser Fall ist auch deshalb von Interesse, weil ausser der Hämatometra auch eine bedeutende Hämatosalpinx derselben Seite vorhanden war, derenEntstehung stets an den Verschluss des Tubenpavillons geknüpft ist.

Bei Durcharbeitung der Literatur fällt es auf, was für Widersprüchen man bei der Erklärung der Aetiologie der Hämatosalpinx begegnet.

Nagel (31) und Veit (51), die in neuerer Zeit diese Frage wieder einer eingehenden Besprechung unterzogen haben, behaupten, „dass das Vorhandensein einer Hämatosalpinx das Kriterium für die extrauterine Acquisition des jeweiligen Genitalverschlusses bildet". Die Entscheidung dieser Frage nach der einen oder anderen Seite hängt lediglich von der Auffassung über die Entstehung des abdominalen Tubenverschlusses ab. Veit (l. c.) und die Anhänger seiner Lehre behaupten, dass der abdominale Tubenverschluss auf keine andere Weise zu erklären sei, als durch bakteriell-entzündliche Vorgänge, und dass, wo eine Hämatosalpinx sich mit einer Gynatresie combinirt, beide Anomalien durch das nämliche entzündlich-infectiöse Agens entstanden sind.

Dem gegenüber betont Mainzer, der einen entsprechenden Fall aus der Landau'schen Klinik anführt, dass der Verschluss der Tube nicht nothwendigerweise durch infectiös-entzündliche Processe erfolgt; „er kann allein durch die productiven Vorgänge der Blutorganisation von Seiten der Tubenwandungen oder des Beckenbauchfells bewirkt werden". Diese Organisation betrifft diejenigen Blutmassen, die bei Gynatresien jeglichen Sitzes und jeglichen Ursprungs so häufig in und aus den Tuben, eventuell auch aus dem Beckenbauchfell erfolgen und ist ganz analog der Bildung peritonealer Pseudomembranen bei intraperitonealen Blutungen infolge Abort oder Ruptur einer ectopischen Gravidität oder überhaupt der Blutorganisation an anderen Körperstellen.

Vor allen Dingen ist doch durch die V eit-Nagel'sche Theorie bei vielen Formen von doppelten Genitalien, wie z. B. in dem uns vorliegenden, wo die Atresie sicherlich congenitalen Ursprungs war und das verschlossene Horn in keiner Communication mit der 
Aussenwelt steht oder je gestanden hat, das Vorkommen der Hämatosalpinx nicht zu erklären.

Allerdings ist Küstner (20) der Ansicht, dass bei den klinisch sich als atretisch erweisenden Nebenhörnern eine sehr enge und deshalb eben nicht nachweisbare Communication nach dem wegsamen Theile des Uterus bestehen kann. Es handelt sich nach Küstner's Ansicht niemals um Atresie, sondern um hochgradige Stenose. Durch diese stenosirte Partie können Keime in die Hämatometra eindringen und eine Infection erzeugen, welche zu dem für die Entstehung einer Hämatosalpinx nothwendigen Verschlusse führt.

Dieser Ansicht widersprechen die Thatsachen, dass, wie in unserem Falle, durch genaueste mikroskopische Untersuchungen nirgends in dem Verbindungsstrang ein Lumen gefunden wurde.

R. Meyer (30), ein Anhänger der Veit-Nagel'schen Theorie, versucht auch hier diese Fälle von einseitiger Gynatresie doppelter Genitalien die Theorie zu halten, indem er hier den Verschluss des Ostium abdominale der Tube durch eine Infection von der offenen Genitalhälfte zu erklären sucht. - Warum jedoch die Infectionserreger durch den gesunden Tubenpavillon hindurchwandern, ohne einen Verschluss zu bedingen, und zum ersten Male in Thätigkeit treten, wenn sie die Tube der atretischen Seite erreichen, ist mir nicht recht fassbar. Mit Recht bemerkt Stratz (44) bierzu: "Welche Verschmitztheit muss man für Infectionskeime annehmen, die alle - diese Theile (in seinem Falle sind Uterus, Tube und das sie umgebende Peritoneum völlig intact) intact lassend, sich endlich "zur der anderen Seite einen Weg bahnen, um hier der Theorie zu Liebe ihrem verderblichen Wesen die Zügel schiessen zu lassen."

Es zeigt also unsere vorliegende Beobachtung auch, dass keineswegs bei der Combination von Gynatresie und Hämatosalpinx letztere gesetzmässig die entzündlich-infectiöse Natur der ersteren beweist.

Endlich können wir auch der Meinung Veit's, der das Blut in der Hämatosalpinx für das Product einer bakteriellen Entzündung der Tube hält, nicht beipflichten.

Es ist nicht zu leugnen, dass gelegentlich ebenso wie in anderen Organen eine infectiöse Entzündung der Tube eine hämorrhagische sein kann. Es ist das jedoch nach den reichen klinischen Erfahrungen sicher eine Ausnahme, ausserdem haben eingehende 
anatomische Untersuchungen gezeigt, dass die schweren entzündlichen Veränderungen gänzlich fehlen (Praetorius, LandauRheinstein). - Alle Autoren, die diesbezügliche Untersuchungen vorgenommen haben, kommen zu dem Resultat, welches wir in unserem Falle haben, dass die Tubenschleimhaut wohl entzündliche Veränderungen aufweist, aber in keinem Falle die schweren, "das normale Structurbild der Tubenschleimhaut bis zur Unkenntlichkeit umwandelnden Zerstörungen", wie z. B. bei der gonorrhoischen Salpingitis und ihren Folgezuständen (Fleck). Da andererseits die Thatsache feststeht, dass in einer grossen Anzahl der Fälle von Gynatresien der Tubenverschluss sicher ohne Infection zu Stande kommt, spricht nichts mit Sicherheit für die infectiöse Natur der intratubären Blutansammlung.

Nach der Meinung Mainzer's besteht überhaupt kein grundsätzlicher Unterschied zwischen den Fällen mit und ohne Hämatosalpinx. Er glaubt, dass die Frage, ob bei einer Gynatresie eine Hämatosalpinx sich bilden wird oder nicht, wahrscheinlich von anderen Factoren abhängt, als von der Aetiologie der Verschlussbildung. Zur Erörterung dieser Verhältnisse muss man sich zunächst einmal klar machen, wie überhaupt die Blutansammlung in der Tube zu Stande kommt. Die einfachste Erklärung für dieses Vorkommen giebt die sogenannte Reffux-Theorie, die bereits 1870 von Olshausen (32) ausgesprochen wurde. Dieselbe nimmt an, dass bei Behinderung des Abflusses das Menstrualblut aus dem Uterus in die Tuben überfliesst und dort nach erfolgtem Verschluss des Pavillons eine Retentionsgeschwulst bildet, vielleicht mit activer Hülfe von Uteruscontractionen (Sänger's „Antiperistaltik", S. 59).

Engström hält diese Theorie vom Reflex für generell unhaltbar. Er hebt hervor, dass die Ansammlung des Blutes immer vorzugsweise, oft ausschliesslich die Pars ampullaris beträfe, während die Pars uterina tubae in solchen Fällen in der Regel gar nicht verändert, ja sogar geschlossen sein könne, und dass Blut in den Tuben bei Gynatresien obne Hämatometra (22) oder auch ohne Gynatresie und Hämatometra, z. B. bei Myomen vorkomme.

Ein anderes gewichtiges Beweismoment dafür, dass der Reflex bei der Entstehung der Hämatosalpingen bei Gynatresien zum mindesten keine regelmässige Rolle bei Gynatresien spielt, liegt 
darin, dass es keineswegs allein das Lumen der Tube ist, wo man bei gynatretischen Verschlüssen Blutansammlungen beobachtet. Es finden sich ausser im Tubenlumen oft schon nach kurzer Dauer der menstruellen Retention Extravasationen auch in der Tubenwand selbst, und zwar nicht nur in der Schleimhaut, sondern auch im Myo- und Perisalpingium.

Ferner erwähnen Engström und Landau-Rheinstein Fälle, wo es sich um Blutungen ins Ovarium, um Blutungen zwischen den Lamellen des Netzes handelt; es giebt Fälle, wo subperitoneale Blutungen (unter dem Beckenbauchfell) oder auch intraperitoneale vorhanden waren, kurz und gut Blutungen an Stellen, die sicher nicht für einen Reflux aus dem Uterus erreichbar waren.

Mikroskopisch finden sich (siehe auch meinen Fall) theils mehr oder weniger frische rothe Blutkörperchen, theils extra- oder intracellulär schollige, grob- oder auch sehr feinkörnige Pigmentmassen (Landau-Pick [22]).

Gründe, die von anderer Seite gegen die Refluxtheorie angeführt sind, die entgegengesetzte Richtung der Tubenperistaltik (Alberts) und die Annahme Hagen's, dass am Ostium uterinum ein ventilartiger Abschluss durch Falten der Uterusschleimhaut stattfindet, scheinen gegenüber den von mir angeführten Gründen von geringerer Bedeutung zu sein.

Es lassen also diese Beobachtungen am gesammten inneren Genitale Gynatretischer keinen Zweifel darüber - zu diesem Schluss kommen wir mit Landau-Pick —, „dass der Inhalt der Hämatosalpinx, so gut wie das Blut in der Tubenwand selbst, den eigenen Gefässen der Salpinx entstammt und dass auch der, Reflex du sang dans la cavité abdominale à travers les trompes', wenn er überhaupt existirt, eine seltene Ausnahme bildet" (vergl. Engström, S. 220, 216).

Welches aber ist die Ursache für diese autochthonen Blutungen in den Tuben?

Sänger (43) [S. 58] erklärt dieselben durch Cireulationsstörungen, besonders Stauungshyperämien oder nspastische und plötzliche Zusammenziehungen des Uterus", die "ganz allein Rhexis entfernterer Venen veranlassen" können.

Engström (S. 229) hält sie für die „Folge einer allgemeinen, sämmtliche Generationsorgane umfassenden Blutfluxion".

Rauscher (38) erklärt diese Rhexis der Gefässe derart, dass Archiv für Gynäkologie. Bd. 74. H. 2. 
374 Katz, Ueber Blutanhäufungen bei doppelten Genitalien etc.

die habituelle Stauung entzündliche Veränderungen der Gefässwände hervorruft und so eine verminderte Widerstandsfähigkeit derselben bewirkt.

Sicher ist, dass in allen diesen Fällen, wie auch Stratz und Landau-Pick betonen, sich ein chronischer Congestionszustand ausbildet, und die bei jeder Menstruation wiederkehrende Steigerung dieses Congestionszustandes ist die wesentliche Veranlassung der Blutung. In dieser chronischen Congestion sehen Landau-Pick - beim Fehlen infectiös-entzündlicher Aetiologie - die Ursache "der gewaltiger productiven Veränderungen in den Organwandungen", während Stratz auch die "zunehmende Druckatrophie" durch das periodisch gesteigerte Wachsthum der Blutcontenta von innen her für einen wirkenden Factor hält.

Als Ursache der chronischen Congestion erscheint LandauPick am wahrscheinlichsten der vom Uterus etc. ausgehende Reiz. Diese Auffassung wird durch die klinische Erfahrung gestützt, dass auch nach der doppelseitigen Castration die "chronisch peritonitischen" Beschwerden durchaus nicht immer zur Ruhe kommen, wenn noch die Hämatometra existirt. - Allerdings ist das immer noch keine absolut befriedigende Erklärung, denn einmal bestand z. B. im Fall I. Landau-Rheinstein eine chronische Congestion der Tube in allen ihren Schichten, obgleich keine eigentliche Blutretention bestand.

Immerhin erscheint uns diese Erklärung noch als die wahrscheinlicbste; eine genügende Klärung dieser einander scheinbar so widersprechenden Thatsachen vermag ich zur Zeit nicht zu geben, und es ist die Aufgabe weiterer Untersuchungen, diese Erage noch mehr zu klären.

Dass die Tuben also unter pathologischen Verhältnissen Blut absondern können, wird, wie wir gesehen, nirgends bestritten. 'Wie steht es aber mit der Blutabsonderung der Tubenschleimhaut unter normalen Verbältnissen, mit der sogenannten Tubenmenstruation? Eine grosse Anzahl von Autoren tritt für das Bestehen einer Tubenmenstruation, $d$. h. dass die Tubenschleimhaut normaler Weise genau wie der Uterus mitmenstruirt, ein (z. B. Wendeler (53), Landau-Rheinstein). - So behaupten Th. LandauRheinstein, dass die Tuben wenigstens unter bestimmten Umständen menstruirten.

Sie nehmen eine primäre Tubenmenstruation an und zwar lediglich aus dem Grunde, weil in einem der von ihnen angeführten 
Fälle beide Tuben mit Blut gefüllt waren, der Uterus aber, trotzdem seine Schleimhant "so normal war, wie man es selten $\mathrm{zu}$ sehen Gelegenheit hat th kein Blut enthält.

Stratz, der auf die Landau-Rheinstein'sche Arbeit ausführlich eingeht, wendet dagegen ein, dass die Hämatometra vielleicht zu wiederholten Malen ihren Inhalt entleert hat und dass die Anwesenheit von Blut in den Tuben unter pathologischen Verhältnissen, wie sie doch in den Fällen von Landau-Rheinstein vorlagen, schliesslich niemals als Beweis dienen kann für eine physiologische Blutabscheidung aus der Tubenschleimhaut. - In Stratz's Fall fand sich im Gegentheil in der Tube der gesunden Seite trotz bestehender Menstruation keine Spur von Blut.

Auch bei den verschiedenen Autoren, die in neverer Zeit für die Tubenmenstruation eingetreten sind (Frank, Thomson, Wehle) handelt es sich um mehr oder weniger pathologische Verhältnisse, die natürlich zur Entscheidung der Frage ohne Bedeutung sind.

Das Blut nun, das unter pathologischen Verhältnissen sich in der Tube befindet, wie in unserem Fall, und das in der Hauptsache durch den congestiven Zustand zu erklären, ist allein im Stande, adhäsive Peritonitis und den daraus resultirenden Tubenverschluss zu erzeugen, wie bereits Sänger, Mainzer und in neuester Zeit Fleck (6) ausgesprochen haben. Ausserdem bewirken auch die productiven Vorgänge der Blutorganisation von Seiten der Tubenwandungen oder des Beckenbanchfells, wie wir bereits hervorgehoben haben, den Verschluss der Tube in Fällen von Gynatresie.

Festzustellen, wie nun schliesslich die das Ostium abdominale umgebenden und es fixirenden Adhaesionen, und wie die produktiven Vorgänge der Blutorganisation den eigentlichen Verschluss zu Stande zu bringen vermögen, das würde zu weit führen und ist von untergeordneter Bedeutung.

Fassen wir das Resultat unserer Ausführungen über die Haematosalpinx zusammen, so ergiebt sich:

Das Vorhandensein einer Hämatosalpinx bei Gynatresien ist keineswegs ein Kriterium für den erworbenen, entzündlich-infectiösen Charakter der Gynatresie.

Der. Verschluss des abdominalen Tubenendes, der zum Vorhandensein einer Hämatosalpinx nothwendig ist, 
wird erzeugt einmal durch den Reiz des austretenden Blutes auf die Serosa, welcher eine das Ostium abdominale umfassende Adhaesionsbildung zur Folge hat und zweitens durch productive Vorgänge der Blutorganisation ron Seiten der Tubenwandungen oder des Beckenbauchfells.

Der Ursprung des Hämatosalpinxblutes ist nur zum geringen Theil in dem Reflux vom Uterus her za suchen, in der Hauptsache stammt es aus den eigenen Gefässen der Tube. Die wesentliche Veranlassung zu diesen Blutungen liegt in der menstruellen, periodisch wiederkehrenden Steigerung eines chronischen Congestionszustandes, wie er sich in solchen Fällen herausbildet: Ueber das Zustandekommen der chronischen Congestion sind keine erschöpfenden Erklärungen vorhanden.

Schliesslich seien mir noch einige Bemerkungen bezüglich der Therapie bei Gynatresien gestattet.

Die Therapie der Gynatresien ist durchaus keine einheitliche, sondern von den verschiedensten Umständen abhängig. Halban sagt: "Wie kaum bei einer anderen Krankheit ist es nöthig, hier $\mathrm{zu}$ individualisiren und alle Umstände und jedes Detail in Betracht zu ziehen, um die für die Patientin günstigste Behandlung zu wählen.

Hier wie überall in der Chirurgie ist das Princip einer conservativen Behandlung festzuhalten, die Entfernung der kranken Organe und die Erhaltung der gesunden."

Es sind bei Uterus duplex und Blutansammlung in einer Hälfte folgende therapeutische Eingriffe bisher in Anwendung gebracht worden:

1. Einfache Entleerung der Hämatometra von der Scheide aus.

2. Entleerung von der Scheide aus mit vorausgehender oder nachfolgender Koeliotomie und Abtragung der. Hämatosalpinx (sammt Ovarium).

3. Koeliotomie, Incision der Hämatometra und Resection bezw. Ausschneidung ihrer Wandung und Naht (Werth).

4. Abtragung des Tubensackes, sowie eines Theiles der ausgedehnten Gebärmutterbälfte mit Einnähen der Sackwandung in die Bauchdecken. 
5. Entfernung der beiderseitigen Gebärmutteranhänge.

6. Supravaginale Amputation der Gebärmutter.

7. Exstirpation der ausgedehnten Gebärmutterhälfte (event. nebst Abtragen der zugehörigen Adnexe im Fall des Bestehens einer Hämatosalpinx).

Mit wenigen Ausnahmen sind die Gynäkologen sich jetzt darüber einig, alle Fälle von Gynatresien, in denen eine Hämatosalpinx nicht mit Sicherheit auszuschliessen ist, durch Laparotomie zu behandeln. Wenn auch. Fritseh der Meinung ist, dass nach seiner Erfahrung die Gefahr des Platzens der Hämatosalpinx stark übertrieben wird, und er spontanes Platzen bei vorsichtiger Palpation nie gesehen hat, so haben in neuerer Zeit besonders Rose auf die Gefahr der Hämatosalpinx und auf die häufige Zerreissung des Tubensackes hingewiesen -- es wurde von allen Autoren (Solowieff, Hegar, Sänger, Veit u. a.) die Exstirpation als nothwendig erkannt. - Ausserdem dürfte auch das Bestehen einer Doppelmissbildung an und für sich schon eine Laparotomie bedingen. Es wird da die Exstirpation der Hämatometra bei Erhaltung des übrigen Genitales (Semiamputatio uteri (Sänger, Wertb) in Frage kommen. Natürlich ist die Exstirpation der durch Menstrualblut verschlossenen Hälfte nur dann vorzunehmen, wenn die nicht verschlossene Hälfte des Uterus functionstüchtig ist, wie z. B. bei unserem Falle.

Im ganzen ist von den von uns angeführten 16 Fällen von Hämatometra im Nebenhorn eines Uterus bicornis 12 mal die Laparotomie ausgeführt worden und $4 \mathrm{mal}$ ist eine andere Behandlungsart eingeschlagen worden, (Fall 1, der bei der Section beobachtet wurde, kommt allerdings nicht in Betracht), 7 mal war bei diesen 12 Fällen ausser der Hämatometra auch eine Hämatosalpinx vorhanden.

Die Anzahl der Laparotomien, die bei doppelten Genitalien bei Verschluss einer Hälfte, mit Vorhandensein einer Hämatosalpinx ausgeführt wurden, ist noch keine sehr bedeutende. Während E. v. Meyer im. Jahre 1893 erst 4 Fälle zusammenstellen konnte, hat Mainzer 1899 bereits 14 Fälle mitgetheilt.

In nevester Zeit sind noch einige Fälle hinzugekommen, so dass es wohl angezeigt erscheint, diese verhältnissmässig seltenen Fälle wieder zusammenzustellen. 


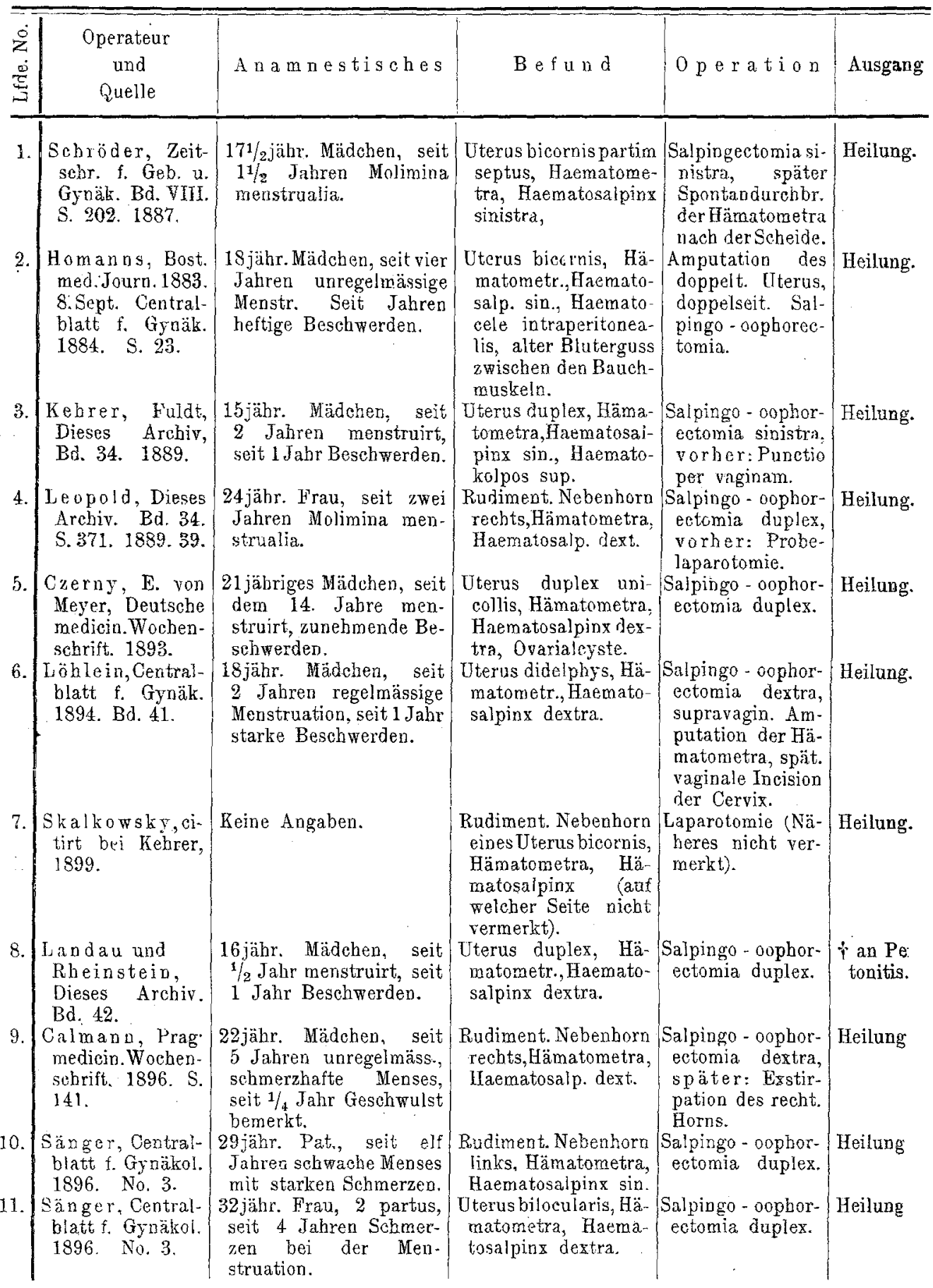


Katz, Ueber Blutanhäufungen bei doppelten Genitalien ete.

\begin{tabular}{|c|c|c|c|c|c|}
\hline 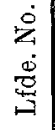 & $\begin{array}{l}\text { Operateur } \\
\text { und } \\
\text { Quelle }\end{array}$ & Anamestisches & $B \in f u n d$ & operation & Ausgang \\
\hline 12. & $\begin{array}{l}\text { Rossa, Centralbl. } \\
\text { f. Gynäkol. } 1896 . \\
\text { 2. No. } 6 .\end{array}$ & $\begin{array}{l}29 \text { jähr. Frau, } 1 \text { Partus, } \\
1 \text { Abort, seit } 1 \text { Jabr } \\
\text { Dysmenorrboe, seitneun } \\
\text { Jabren Geschwulst im } \\
\text { Leib bemerkt. }\end{array}$ & $\begin{array}{l}\text { Rudiment. Nebenhorn } \\
\text { links, Hämatometra, } \\
\text { Haematosalpinx sin. }\end{array}$ & $\begin{array}{l}\text { Exstirpation des } \\
\text { Uterushorns und } \\
\text { der Hämatosalp. }\end{array}$ & Heilung. \\
\hline 13. & $\begin{array}{l}\text { Kézmársky, } \\
\text { Bäcker, Central- } \\
\text { blatt f. Gynäkol. } \\
1896 . \quad \text { S. } 883 .\end{array}$ & $\begin{array}{l}\text { 24jähr. Frau, } 4 \text { Partus, } \\
\text { seit } 5 \text { Jabren Geschwulst } \\
\text { im Leib bemerkt. }\end{array}$ & $\begin{array}{l}\text { Uterus duplex (nicbt } \\
\text { didelphys), Häma- } \\
\text { tometra, Haemato- } \\
\text { salpinx sio. }\end{array}$ & $\begin{array}{l}\text { Salpingectomia si- } \\
\text { nistra, A mputat. } \\
\text { derHämatometra } \\
\text { und Einnäben des } \\
\text { Sacks. }\end{array}$ & fimshocl \\
\hline 14. & $\begin{array}{l}\text { Kleinhaus, Ma- } \\
\text { natsschr. f. Geb. } \\
\text { u. Gynäk. Bd.V. }\end{array}$ & $\begin{array}{l}\text { 17jahr. Mädehen, seit } \\
11 / 2 \text { Jabren Menses, seit } \\
3 \text { Monaten starke Be- } \\
\text { schwerden. }\end{array}$ & $\begin{array}{l}\text { Uterus duplex, Hä- } \\
\text { matometra, Haema- } \\
\text { tosalpinx sinistra. }\end{array}$ & $\begin{array}{l}\text { Salpingo - oophor- } \\
\text { ectornia sinistra, } \\
\text { vorher: Punctio } \\
\text { per vaginam. }\end{array}$ & Heilung. \\
\hline 15. & $\begin{array}{l}\text { Land a u, Mainzer, } \\
\text { Dieses Archiv. } \\
\text { Bd. } 57 .\end{array}$ & $\begin{array}{l}15 \text { jähr. Mädchen, seit } \\
1 / 2 \text { Jahr Menses mit } \\
\text { starken Beschwerden. }\end{array}$ & $\begin{array}{l}\text { Uterus duplex, Hä- } \\
\text { matometra, Haema- } \\
\text { tosalpinx sin. }\end{array}$ & $\begin{array}{l}\text { Salpingo-oophor- } \\
\text { ectomia sinistra. }\end{array}$ & Heilung. \\
\hline 16. & $\begin{array}{l}\text { Preetorius, Ex- } \\
\text { trait des Annales } \\
\text { de la Société mé- } \\
\text { dico-cbirurgicale } \\
\text { d'Anvers. } 1899 \text {. } \\
\text { Cit. b. Stratz, } \\
\text { Zeitschr. f. Geb. } \\
\text { u. Gynäk. Bd. } 45 \text {. } \\
\text { S. } 4 \text {. }\end{array}$ & $\begin{array}{l}\text { 1Sjäbr. Mädchen, seit } \\
\text { dem 11. Jahre regel- } \\
\text { mässige Menses, seit } \\
\text { 2 Jahren starke Schmer- } \\
\text { zen, die seit } 3 \text { Monaten } \\
\text { unerträglich. }\end{array}$ & $\begin{array}{l}\text { Uterus duplex, Vagina } \\
\text { dupl., Hämatometra, } \\
\text { Haematosalpinx sin. } \\
\text { und Haematovarium } \\
\text { sin. }\end{array}$ & $\begin{array}{l}\text { Abdominale Radi- } \\
\text { caloperation. }\end{array}$ & Heilung. \\
\hline 17. & $\begin{array}{l}\text { Stratz, Zeitschr. } \\
\text { f. Geb. u. Gyn. } \\
\text { Bd. } 45 . \text { S. } 15 .\end{array}$ & $\begin{array}{l}15^{1 / 2 j a ̈ h r . ~ M a ̈ d c h e n, ~ s e i t ~} \\
21 / 2 \text { Jahren Mensesregel- } \\
\text { mässig, aber mit star- } \\
\text { ken, stets zunehmenden } \\
\text { Schmerzen. }\end{array}$ & $\begin{array}{l}\text { Uterus duplex, Häma- } \\
\text { tometra, Haemato- } \\
\text { salpinx sin., Haemat- } \\
\text { ovarium sin. }\end{array}$ & $\begin{array}{l}\text { Salpingo-oophor- } \\
\text { ectrimia duplex, } \\
\text { mach Punction d. } \\
\text { Hämatometra per } \\
\text { vaginam. }\end{array}$ & Heilung. \\
\hline 18. & $\begin{array}{l}\text { v. Pauer, Central- } \\
\text { blatt f. Gynäl. } \\
1902 . \text { S. } 660 .\end{array}$ & $\begin{array}{l}\text { 18jähr. Märchen, seit } \\
\text { dem } 17 \text {. Jabre Menses, } \\
\text { stets mit Schmerzen, } \\
\text { in der letzten Zeit auch } \\
\text { in der intermenstruellen } \\
\text { Zeit Schmernen. }\end{array}$ & $\begin{array}{l}\text { Uterus bilocularis, Hä- } \\
\text { matometra, Hämato- } \\
\text { salpinx sin. }\end{array}$ & $\begin{array}{l}\text { Salpingo - oophor- } \\
\text { ectomia sinistra, } \\
\text { Abtragen der Hä- } \\
\text { matometra. }\end{array}$ & Heilung. \\
\hline 19. & A bel. & $\begin{array}{l}26 \text { jär. Mádehen, mit dem } \\
\text { 18. Jahre Menses, mit } \\
\text { heftigen Scbmerzen. }\end{array}$ & $\begin{array}{l}\text { Rudiment, Nebenhorn } \\
\text { eines Uterus bicornis } \\
\text { unicollis, Hämato- } \\
\text { metra, Haematosalp. } \\
\text { dextra. }\end{array}$ & $\begin{array}{l}\text { Vaginale Exstir- } \\
\text { pation d. Häma- } \\
\text { tometra u. Sal- } \\
\text { pingo-oophorec- } \\
\text { tomia dextra. }\end{array}$ & Heilung. \\
\hline
\end{tabular}

Im Ganzen finden wir also, einschliesslich des von mir beschriebenen, 19. Fälle, in welchen Gynatresien bei doppeiten Genitalien bej Vorhandensein von Hämatosalpinx dureh Laparotomie bebandelt wurden.

Dazu kommen noch 5 Fälle (No. 8, 12, 13, 15, 16 der Tabelle I) von Hämatometra im rudimentären Nebenhorn ohne Hämatosalpinx und ausserdem 4 Fälle von doppeltem Uterus mit Hämato- 
metra (ebenfalls ohne Vorhandensein von Hämatosalpinx) - Fall Werth, Zillessen-Schultze, Fall I Halban's, Opitz') -, bei denen die Laparotomie zur Ausführung kam - im Ganzen also sind es 28 Fälle, bei welchen bei Gynatresien am doppelten Uterus laparotomirt wurde.

Das eingeschlagene Operationsverfahren war also, wie wir sehen, keineswegs in allen den Fällen dasselbe.

Fragen wir uns nun nach der rationellsten Art des Vorgehens, so ist unter allen Umständen, wie wir schon oben auseinandergesetzt, zur Klarlegung der Verhältnisse eine primäre Laparotomie geboten, auch wenn keine Hämatosalpinx vorhanden ist. Wagner hat 189643 Fälle von Haematometra unilateralis gesammelt, von welchen 6 unoperirt geblieben sind, bei 26 wurde Punction oder Incision von der Scheide aus gemacht und in 11 Fällen hat man die Koeliotomie ausgeführt. Es sind von den 43 Fällen gestorben: 5 unoperirt gebliebene, 10 nach der Punction event. Incision; geheilt: unoperirt $1(?)$, nach der Punction event. Incision 16, nach der Koeliotomie 11. - Obgleich diese Statistik absolut nicht vollständig ist, sehen wir doch, dass die Koeliotomie bei der relativen Ungefährlichkeit derselben bessere Resultate lieferte, als die im Dunkeln ausgeführte Operation von der Scheide aus.

In meiner Statistik, in der allerdings auch die Fälle berücksichtigt sind, in denen Hämatosalpinx bestand, haben wir in den 28 Fällen 2 'Todesfälle zu verzeichnen.

Im Einzelnen haben wir nun folgende Möglichkeiten zu beachten:

1. Fälle von Haematometra unilateralis ohne Hämatosalpinx.

Man wird also bei diesen Fällen zunächst eine Laparotomie, einerlei, ob der Tumor vor der Operation als Hämatometra oder Myom oder als Kystom oder als sonstiger Adnextumor diagnosticirt wurde $^{2}$ ) vornehmen. - Es diente hier die Laparotomie, wie schon erwähnt, in vielen Fällen nur zur Orientirung und es wurde dann von der Scheide aus zur Oeffnung der Hämatometra geschritten

1) Bei Werth, Zillessen u. Opitz handelte es sich um Ausschälung des Sackes bei Uterus septus bilocularis, ein Fall Halban um eine abdominale Implantation des Fundus uteri in die Vagina.

2) Cf. die Fälle 13 und 16 der Tabelle I. 
oder es wurde Koeliotomie mit Einnähung des Blutsackes in die Bauchwunde gemacht.

Bei Hämatometra im Nebenhorn genügt z. B. bei dünnem Stiel die einfache Abbindung, dieselbe wurde jedoch auch vielfach bei ziemlich inniger Verbindung der Hämatometra mit dem anderen Uterus ausgeführt.

Im Ganzen wurde in 4 Fällen $^{1}$ ) (meinen Fall mit eingerechnet) bei Hämatometra im rudimentären Nebenhorn und in 3 Fällen bei Hämatometra bei Uterus bilocularis ${ }^{2}$ ) die Exstirpation der Hämatometra bei Erhaltung des übrigen Genitales ausgeführt. Natürlich kommt diese Operation, wie schon erwähnt, nur dann in Frage, wenn die andere nicht verschlossene-Hälfte des Uterus functionstüchtig ist.

In einem Fall (cf. No. 16 der Tabelle I) wurde wegen der technischen Unmöglichkeit - „bei der breitbasigen Verbindung der beiden Uterushälften war eine Exstirpation der Hämatometra schwer. ausführbar" - die Totalexstirpation ausgeführt (die rechten Adnexe, die "degenerirt und atrophisch waren", wurden mitentfernt.)

2. Fälle von Haematometra unilateralis mit der Complication einer Hämatosalpinx.

Im Einzelnen haben wir hier folgende Möglichkeiten zu unterscheiden:

a) Finden wir bei der Laparotomie auch die Tube der anderen Seite oder das Ovarium der anderen Seite derartig erkrankt, dass ihre Zurücklassung entweder eine directe Lebensgefahr darstellen würde, oder wenigstèns keine vollständige Genesung ermöglichte, so ist die doppelseitige Adnexexstirpation angezeigt. Die Hämatometra kann ausser Acht gelassen werden, da sie sich erfahrungsgemäss nach der Castration zurückbildet.

Allerdings scheint nach $\mathrm{M}$ ainzer doppelseitige Hämatosalpinx viel seltener bei doppeltem Genitalcanale als bei einfachem vorzukommen. Es ist bisher bei doppeltem Genitalcanale noch niemals wegen doppelseitiger Hämatosalpinx die Castration ausgeführt worden.

Sind jedoch die Adnexe der anderen Seite vollständig gesund, so begnügten sich einige Operateure mit der Exstirpation des

1) Die Fälle 8, 12, 13, 15 der Tabelle I.

2) Die Fälle Werth (54), Zillessen (57), Opitz (33). 
Hämatosalpinx und des zugehörigen Ovariums, ohne die Hämatometra in Angriff zu nehmen (Schröder, Landau u. A.); doch musste manchmal durch eine zweite Operation die Hämatometra behoben werden (Calmann u. A.), da nach der ersten Operation die Menstruation andauerte und die Hämatometra weiter zunehmen konnte.

Dann wurde in vielen Fällen auch die gesunde Seite, das gesunde Ovarium bez. Ovarjum und Tube mitentfernt, um dadurch die Menstruation zum Stillstand zu bringen und die secundäre Atrophie des Horns zu ermöglichen. Von diesem Gesichtspunkte aus haben auch Homanns (14), Leopold (23), Czerny (28), L. Jandau (22), Sänger (43), Stratz (44) die doppelseitige Adnexexstirpation ausgeführt.

Es fragt sich jedoch, ob wir nicht verpflichtet sind, primär ein mehr conservatives Verfahren einzuschlagen als die Castration, umsomehr, als es sich hier immer um ziemlich jugendliche Individuen handelt und eine derartige Verstümmelung nicht am Platze ist. Das ideale Verfahren ist darum, wie schon Sänger (43) betont, die Wegnahme der atretischen Seite mit Erhaltung der gesunden Seite. Alle Operateure betonen hierbei die ausserordentlich grosse Schwierigkeit, den atretischen Uterus mit zu entfernen, und es ist bis jetzt nur in wenigen Fällen gelungen, das atretische Nebenhorn bei der Operation von den Bauchdecken aus mit zu entfernen.

Interessant ist es, was Sänger (43, S. 48), der 2 diesbezügliche Fälle veröffentlicht hat, speciell über diese Operation in dem einen Falle, den er operirt hat, sagt:

"Es ging nicht an, hier conservativ zu verfahren, in der Weise, dass etwa nach Entfernung nur der rechtsseitigen Adnexe, Ausbülsung und Erhaltung der linken Adnexe die versehlossene rechte Uterushälfte geöffnet, entleert und in die Bauchwunde eingenäht wurde, wie dies wiederholt bei grossen Hämatometra- und auch Hämatosalpinxsäcken gemacht worden ist: es war durchaus unmöglich, den Doppeluterus der vorderen Bauchwand nahe genug zu bringen, um dies ausführen zu köunen. Ebenso wenig konnte daran gedacht werden, die rechte Uterushälfte mitsammt zugehörigen Adnexen fortzunehmen. Man braucht sich nur zu überlegen, dass die Absetzung in oder entlang der rechten seitlichen Wand der zu erhaltenden Uterushälfte stattfinden musste, deren Hauptgefässe doch nicht unterbunden werden konnten, um zu er- 
messen, dass auf eine sichere Blutstillung nebst guter Deckung der breiten und langen Wunde kaum zu rechnen war."

Natürlich gelingt es in den Fällen, wo die verschlossene Uterushälfte mit der offenen in gar keinem engen Zusammenhange oder gar getrennt ist, wie z. B. bei einem gestielt aufsitzenden Nebenhorn des Uterus oder bei einem Uterus dipelphys, mit Leichtigkeit, die Tuben- und Uterusgeschwulst zu entfernen.

So hat Löhlein (24) bei einem Uterus didelphys und Verschluss der recbten Seite die Adnexe entfernt und den atretischen Uterus supravaginal amputirt (allerdings musste späterhin noch die Cervix vaginal incidirt werden).

Anders dagegen steht die Sache in den Fällen, wo z. B. das atretische Nebenhorn mit der offenen Uterushälfte in sehr innigem Zusammenhang steht, wie z. B. in dem von mir beschriebenen Falle. Jedenfalls müssen wir auch in diesen Fällen zum mindesten versuchen, das conservative Verfahren einzuschlagen, die Exstirpation des atretischen Horns bez. der zugehörigen Hämatosalpinx.

Freilich wird hier von den meisten der Einwand erhoben werden, dass die technische Ausführung unmöglich und nicht ohne grosse Gefahr für die Patientin ist. Trotzdem dürften in den meisten Fällen die technischen Schwierigkeiten zu überwinden scin und was die Gefahr einer Infection betrifft, so kann sie bei einem streng aseptischen Verfahren auch kaum grösser werden. - Auch in unserem Falle war es äusserst schwierig, das atretische Nebenhorn, das durch musculöse und bindegewebige Verbindung mit dem Uterus zusammenhing, abzusetzen, es gelang jedoch Herrn Dr. Abel, den Uterus successive von Fundus anfangend, mit ein paar grossen Nähten abzubinden and dann abzuschneiden. Es wurde darauf versucht die Tube, das Lig. infundibulo-pelvicum und ebenso das Lig. latum abzuschneiden; man musste hier bis in die Vagina gehen, und die A. uterina dieser Seite mit umstechen.

Im Ganzen ist die Zahl der Fälle, wo bei doppeltem Uterus nur der atretische Theil ev. bei Bestehen einer Hämatosalpinx mit Wegnahme der Adnexe der atretischen Seite entfernt wurde, keine sehr zahlreiche.

Unseren Fall eingerechnet sind es bei Hämatometra im Nebenhorn eines Uterus bicornis 7 Fälle (Fall 8, 10, 11, 1) 12, 13, 14,

1) Im Fall Calmann wurde allerdings bei der 1. Laparotomie nur eine Salping0-0ophorectomia dextra, und erst bei der 2. sich als nothwendig herausstellenden Laparation wurde die Hämatometra exstirpirt. 
15 der Tabelle I.) ${ }^{1}$ ), ferner Fall Löhlein, der bereits erwähnt, Fall v. Pauer (Fall 18 der Tabelle II.), Werth, ZillessenSchultze, Opitz - also im Ganzen 12 Fälle, in denen es gelang die Hämatometra im atretischen Theile zu exstirpiren.

Ist es technisch absolut nicht möglich, die Exstirpation der atretisehen Seite auszuführen, so ist die Totalexstirpation des ganzen Uterus (bei Bestehen einer Hämatosalpinx mit gleichzeitiger Entfernung der Adnexe) wie sie von Martin, V. Rosenthal und Pinne-Pintor (auf vaginalem Wege) mit gutem Erfolge im Interesse einer rascheren und radicaleren Heilung der Patientin ausgeführt wurde, berechtigt.

Auf jeden Fall aber ist dieselbe der Castration, die eine Atrophie des atretischen Horns bewirken soll, vorzuziehen. Denn abgesehen davon, dass eine Castration sicher nicht ohne bedeutsame Gonsequenzen für das weitere Leben der Frau ist, hat auch die Erfahrung gelehrt, dass in einzelnen Fällen trotz Entfernung beider Ovarien der Stumpf zu stets wiederkehrender Peritonitis Veranlassung gab. So sah sich z. B. Treub genöthigt, nachträglich den Uterus noch zu exstirpiren und in dem von Stratz operirten Falle daverte es trotz der Castration; noch beinahe ein Jahr, ehe alle Beschwerden gewichen waren.

Der von mir beschriebene Fall, in dem also die atretische Uterushälfte nebst Adnexen dieser Seite entfernt wurde, gewinnt auch noch insofern eine gcwisse Bedeutung dadurch, als er durch die vaginale Koeliotomie operirt worden ist. Es ist dies das erste Mal, dass diese Operation auf vaginalem Wege ausgeführt worden ist.

Dass der vaginalen Koeliotomie auf Grund der verschiedensten Statistiken gegenüber der abdominalen, auch bei Berücksichtigung der ncuesten Methoden der Schnittführung und Vernähung bei Laparotomien, natürlich in den dazu geeigneten Fällen, der Vorzug eingeräumt werden muss, steht fest. Die Indicationsgrenze schwankt, wemn das auch viele Autoren absolut nicht zugeben wollen, nach der Ausbildung der Technik des Operateurs.

Es gelang Herrn Dr. Abel ganz leicht, mit Hülfe des vorderen Scheidenlängsschnittes, den er jetzt gewöhnlich bei der vaginalen Koeliotomie anwendet, den Uterus und dann die Hämatometra vor die Vulva zu holen und hierauf in der geschilderten Weise (Ein-

1) Bei Fall $8,12,13,15$ war keine Hämatosalpinx vorhanden. 
stossen der Hämatosalpinx mit der Kornzange) die Operation zu Ende zu führen. - Wenn v. Pauer meint, dass in diesen Fällen der vaginale Weg nur dann berechtigt ist, "wenn die Situation nach minutiöser Diagnose ganz klar da steht, und wenn man sicher sein kann, nicht auf die Laparotomie übergehen zu müssen, und wenn nach seiner Ansicht bei Bestehen einer grösseren Hämatosalpinx in allen Fällen das abdominale Verlahren vorzaziehen ist, so ist einmal dagegen einzuwenden, dass es absolut nichts schadet, im Falle der Unmöglichkeit, vaginal die Operation zu Ende zu führen, die abdominale Eröffnung anzuschliessen. - Im Uebrigen ist nicht recht einzusehen, warum bei Vorhandensein einer grossen Hämatosalpinx nicht vaginal operirt werden soll. - Es bestehen doch da diesselben Verhältnisse wie z. B. bei grösserem cystischen beweglichen Ovarialtumor, wo die vaginale Operation in allen Fällen Anwendung findet, in denen man glaubt, dass die Hervorleitung der Cyste, entweder in toto oder nach Entleerung des Inhaltes möglich ist. -

Die Patientin hat, wie wir gesehen, eine glatte Reconvalescenz durchgemacht und konnte gesund entlassen werden.

In allerneuester Zeit empfiehlt Pinne-Pintor als bestes Verfahren den vaginalen Operationsweg (insbesondere den vorderen Scheidenschnitt). - Allerdings war es ihm bei der breitbasigen Verbindung der beiden Uterushälften nicht möglich, die Exstirpation der Hämatometra auszuführen; sondern er machte mittelst vorderer Kolpotomie (Krenzschnitt) die Totalexstirpation des Uterus mit gleichzeitiger Wegnahme der Adnexe der atretischen Seite.

In unserem Falle gelang es, auf dem Wege der vaginalen Koeliotomie in verhältnissmässig ungefährlicher Weise der Forderung Sänger's die Wegnahme der verschlossenen, blutgefüllten Uterushälfte und der zugehörigen Adnexe bei Erhaltung der gesunden Adnexe und des Uterus, nachzukommen.

Wenn auch, wie Calmann betont, bei der Entscheidung des einzuschlagenden Verfahrens äussere materielle und sociale Verhältnisse in Betracht gezogen werden müssen ${ }^{1}$ ), so müssen wir jedoch von rein ärztlichem Standpunkte das Verfahren als das idealste ansehen, welches dem Körper seine Functionen ertheilt und zugleich die Patientin von Lebensgefahr und Beschwerden

1) Der Patientin E. v. Meyer's (28), die 6 Geschwister hatte, kam es mehr darauf an, arbeitsfähig als zeugungsfähig zu sein. 
befreit. - So wurde auch in unserem Falle, das conservativste und zugleich radicalste Verfahren gewählt, das der Patientin die Möglichkeit einer Conception im zurückgebliebenen, vollkommen normalen Uterus gelassen und die Geschlechtsorgane in einen functions/ähigen Zustand zurückversetzt hat.

Zum Schlusse meiner Arbeit sei es mir gestattet, meinem hochverehrten Chef Herrn Dr. Abel für die freundliche Anregung zu dieser Arbeit und die hülfreiche Förderung derselben meinen verbindlichsten Dank auszusprechen. --

\section{L i t e r a t ur.}

1. A bel, K., Fall von Hämatometra im rechten atretischen Nebenhorn eines Uterus duplex mit Hämatosalpinx. Operation. Ileilung. Berliner klinische Wochenschr. 1901. No. 51.

2. Boije, 0. A., Ueber Hämatometra im rudimentären Horne eines Uterus bicornis unicollis. Mittheil. aus der gynäkologisehen Klinik des Prof. Dr. O. Engström (Helsingfors). Bd. III. 1901.

3. Bond, Die Menstruation der Tuben. Brit. med. journ. 1898.

4. Calmann, Ueber einen Fall von Hämatometra im rudimentären Horn. Prager med. Wochenschr. 1896.

5. Engström, 0., Zur Kenntniss und Behandlung der nicht puerperalen Gynatresien mit consecutiver Retention bei einfachem Uterusvaginalcanal. Mittheil. aus der gynäkolog. Klinik des Prof. Dr. Engström (Helsingfors). Bd. II. Heft 3. 1899.

6. Fleck, G., Die Entstehung des Tubenverschlussos bei der gynatretischen Hämatosalpinx. Dieses Archiv. Bd. 71. H. 2.

7. Fritseb, H., Die Krankheiten der Frauen. 4. Aufl. 1889.

8. Fürst, L., Bildungshemmung des Utero-vaginalcanals. Monatsschr. für Geburtskunde u. Frauenkrankheiten. Berlin. 1867. Bd. 30 . Heft 2 น. 3.

9. Fuld, Salpingotomie wegen Hämatosalpinx. Dieses Arch. 1889.

10. Frank, Centralblatt für Gynäk. 1901. S. 720.

11. Gebbard, Pathologische Anatomie der weiblichen Sexualorgane. Leipzig. 1899.

12. Halban, Zur Therapio der Gynatresien. Zeitschr. f. Geburtsh. u. Gyn. Bd. 49. 1. 1903.

13. Hegar, A., Die Hämatometra in dem rudimentäron Horne des Uterus unicornis. Beiträge zur Geburtshülfe u. Gynäkol. Bd. IlI. Berlin. 18ä4.

14. Homans, J, Uterus bicornis, Hämatometra, supravaginale Amputation, Heilung. Boston. med. and surgical journal. 8. Septbr. 1883. Cit. nach Centralblatt für Gynäk. 1884. S. 23.

15. Kehrer, A., Ueber Gynatresie und deren Behandlung. Frauenarzt. Bd.7. 1892. 
16. Kehrer, F., Das Nebenhorn des doppelten Uterus. In.-Dissert. Heidelberg. 1899.

17. Kleinhans, Ueber einen Fall von Hämatometra lateralis mit Hämatosalpinx. Monatsschrift f. Geburtsh. u. Gyn. Bd. V. 1897.

18. Kézmárszky, Citirt bei Bäker: Uterus didelphys, Hämatometra urıd -salpinx lat. sin. Centralblatt f. Gyn. 1896. S. 883.

19. Kussmaul, Von dem. Mangel, der Verkümmerung und Verdoppelung der Gebärmutter. Würzburg. 1859 .

20. Küstner, Lehrbuch der Gynäkologie.

21. Landau, Th. u. Rheinstein, Ueber das Verhalten der Schleimhäute in verschlossenen $\mathrm{u}$. missbildeten Genitalien und über die T'ubenmenstruation. Dieses Archiv. 1892.

22. Landau, L. und Pick, Ueber die mesonephrische Atresie der Müllerschen Gänge, zugleich ein Beitrag zur Lehre von den mesonephrischen Adenomyomen des Weibes und zur Klinik der Gynatresien. Dieses Arch. Band 64.

23. Leopold, Ueber Blutansammlung im verschlossenen Utero-vaginalcanal und die Salpingotomie. Dieses Arch. Bd. 36. 1889.

24. Löhlein, Uterus didelphys, Hämatometra et Haeroatosalpinx sinistri lateris: Operation. Centralbl. f. Gynäk. 1894. S. 997.

25. Mainzer, Zur Aetiologie und Therapie der Gynatresien, insbesondere der Gynatresien. Dieses Arch. 1899.

26. Martin, Krankheiten der Eileiter. Leipzig. 1895.

27. Mangiagalli, E., Tre casi di utero unicorne con come rudimentale (osservazione II). Annali die Ostetrica e Ginecologia. Cit. bei B o ije. 1892.

28. v. Meyer, E., Casuistischer Beitrag zur operativen Behandlung der in Folge von Gynatresien auftretenden Hämatosalpinx mit besonderer Berücksichtigung der Salpingotomie. Deutsche med. Wochenschr. 1893. No. 39. Seite 936.

29. Meyer. H., Ueber die Verdoppelungen der Gebärmutter und ihre Complicationen mit Schwangerschaft und einseitiger Atresie. In.-Diss. Züriçh. 1883. S. 38 .

30. Meyer, Rob., Zur Aetiologie der Gynatresien auf Grund der einschlägigen Casuistik. Zeitschr. f. Geburtsh. u. Gynäk. Bd. 34. 1896 u. Bd. 36. 1897.

31. Nagel, Zur Lehre von der Atresie der weiblichen Genitalien. Zeitschr. f. Geburtsh. u. Gynäkol. Bd.34. 1096. (Vergl. aueb in Veit's Handbuch der Gynäkologie. Bd. I. 1897.)

32. Olhausen, Hämatocele und Hämatometra. Dieses Arch. 1870.

33. Opitz, E., Ueber Hämatometra im atretischen Horn eines Uterus bilocularis. Zeitschr. f. Geburtsh. u. Gynäk. Bd. 45. 1901. S. 565.

34. v. Pauer, Ein Fall von Uterus duplex separatus. Centralbl. f. Gynäk. 1902. S. 560.

35. Pick, L., Zur Aratomio und Genese der doppelten Gebärmatter. Dieses Arch. Bd. 57. 1899.

36. Pinna-Pintor, A. (Turin), Ein Fall von Hämatometra im rudimentären Nebenhorn eines Uterus bicornis. Giorn. di gin. e de ped. 1902. No. 23. Git. nach Centralkl. f. Gyn. 1904. S. 407. 
$388 \mathrm{Katz}$, Ueber Blutanhäufungen bei doppelten Genitalien etc.

37. Pincus, L., Praktisch wichtige Fragen zur Nagel-Veit'schen Theorie. Sammlung klinischer Vorträge. N. F. No. 229/300.

38. Rausch er, Ueber Hämatosalpinx bei Gynatresien. Zeitschr. f. Geburtsh. u. Gyn. Bd. 49. 1903, S. 417.

39. Rose, Die Operation bei Hämatometra. Monatsschr. f. Geburtskunde u. Franenkrankh. Bd. 29. 1867.

40. Rossa, 2 Fälle von Gynatresien. Centralbl. f. Gyn. 1894. No. 18.

41. Derselbe, Zur operativen Therapie bei Gynatresien. Centralbl. f. Gyn. 1896. No. 6.

42. v. Rosenthal, Hämatometra unilateralis im rechten Horn eines Uterus bicornis. Koeliohysterostomia. Heilung. Monatsschr. f. Geburtsh. u. Gyn. Bd. VIII, 1898.

43. Sänger; M., Drei Fälle von Salpingo-oophorectomia dụplex bei Haematometra gynatretica. Centralbl. f. Gyn. 1897. S. 49.

44. Stratz, Blutanhäufungen bei einfachen und doppelten Genitalien. Zeitschrift f. Geb. u. Gyn. 1901.

45. Skalkowski, Warschau, Przeglad Lekarski. 1894. No. 22-24. Citirt nach E. Kehrer.

46. Solowieff, Zeitsohr. f. Gebh. u. Gyn. Bd. VII. H. 1.

47. Stoltz u. Lobstein, Gazette médicale de Strasbourg. 1860. No. 3. Cit. bei E. Kehrer.

48. Schröder, Sitzung der Gesellschaft für Geburtshülfe und Gynäkologie zu Berlin. Zeitschr. f. Geburtsh. u. Gyn. 1882. Bd. VIII.

49. Thomsen, Zur Frage der Tubenmenstruation. Centralbl, f. Gyn. 1898.

50. Treub-Maasland, Cit. bei Stratz.

51. Veit, Ueber Hämatosalpinx bei Gynatresie. Berliner klin. Wochenschr. 1896. No. 16. (Vergl, auch Veit's Handbuch der Gynäk. Bd. I. 1897.

52. Wehle, Ueber Tubenmenstruation, Sitzungsbericht der gynäkol. Gesellschaft in Dresden. Centralbl. f. Gyn. 1901.

53. Wendeler, in Martin's Krankheiten der Eileiter. Leipzig. 1895.

54. Werth, Dieses Archiv. Bd. 48.

55. v. Winckel, Ueber die Eintheilung, Entstehung und Benennung der Bildungshemmungen der weiblichen Sexualorgane. Volkmann's Sammlung klin. Vorträge. 1899. S. 251/252.

56. Winter, Demonstration in der Geselischaft für Geburtshülfe und Gynäk. zu Berlin. Vergl. Zeitschr. f. Geburtsh. u. Gyn. Bd. 38. 1898.

57. Zillessen, Hämatometra in dem verschlossenen rudimentären Horn eines Uterus bilocularis. Inaug.-Diss. Jena. 1899. 